\title{
BMJ Open Socioeconomic inequalities and adverse pregnancy outcomes in the UK and Republic of Ireland: a systematic review and meta-analysis
}

Katie Thomson (D) , ${ }^{1}$ Malcolm Moffat, ${ }^{1}$ Oluwatomi Arisa, ${ }^{1}$ Amrita Jesurasa, ${ }^{2}$ Catherine Richmond, ${ }^{1}$ Adefisayo Odeniyi, ${ }^{1}$ Clare Bambra, ${ }^{1,3}$ Judith Rankin, ${ }^{1,3}$ Heather Brown, ${ }^{1,3}$ Julie Bishop, ${ }^{4}$ Susan Wing, ${ }^{4}$ Amy McNaughton, ${ }^{4}$ Nicola Heslehurst ${ }^{1,3}$

To cite: Thomson $\mathrm{K}$, Moffat $\mathrm{M}$, Arisa 0 , et al. Socioeconomic inequalities and adverse pregnancy outcomes in the UK and Republic of Ireland: a systematic review and meta-analysis. BMJ Open 2021;11:e042753. doi:10.1136/ bmjopen-2020-042753

- Prepublication history and additional material for this paper are available online. To view these files, please visit the journal online (http://dx.doi. org/10.1136/bmjopen-2020042753).

Received 20 July 2020

Revised 04 January 2021

Accepted 27 January 2021

Check for updates

(C) Author(s) (or their employer(s)) 2021. Re-use permitted under CC BY-NC. No commercial re-use. See rights and permissions. Published by BMJ.

${ }^{1}$ Population Health Sciences Institute, Faculty of Medical Sciences, Newcastle University, Newcastle upon Tyne, UK

${ }^{2}$ Primary Care Division, Public Health Wales, Cardiff, UK

${ }^{3}$ Fuse, The Centre for

Translational Research in Public Health, Newcastle University,

Newcastle upon Tyne, UK

${ }^{4}$ Health Improvement Division,

Public Health Wales, Cardiff, UK

Correspondence to

Nicola Heslehurst;

nicola.heslehurst@ncl.ac.uk

\section{ABSTRACT}

Objective There has been an unprecedented rise in infant mortality associated with deprivation in recent years in the United Kingdom (UK) and Republic of Ireland. A healthy pregnancy can have significant impacts on the life chances of children. The objective of this review was to understand the association between individual-level and householdlevel measures of socioeconomic status and adverse pregnancy outcomes.

Design Systematic review and meta-analysis.

Data sources Nine databases were searched (Medline, Embase, Scopus, ASSIA, CINAHL, PsycINF0, BNI, MIDRIS and Google Scholar) for articles published between 1999 and August 2019. Grey literature searches were also assessed.

Study selection criteria Studies reporting associations between individual-level or household socioeconomic factors on pregnancy outcomes in the UK or Ireland. Results Among the 82353 search results, 53821 titles were identified and 35 unique studies met the eligibility criteria. Outcomes reported were neonatal, perinatal and maternal mortality, preterm birth, birth weight and mode of delivery. Pooled effect sizes were calculated using random-effects meta-analysis. There were significantly increased odds of women from lower levels of occupation/social classes compared with the highest level having stillbirth (OR 1.40, $95 \% \mathrm{Cl} 1.23$ to $1.59, \mathrm{I}^{2} 98.62 \%$ ), neonatal mortality (OR $1.39,95 \% \mathrm{Cl} 1.22$ to $\left.1.57, \mathrm{I}^{2} 97.09 \%\right)$, perinatal mortality (OR $1.39,95 \% \mathrm{Cl} 1.23$ to $1.57, \mathrm{I}^{2} 98.69 \%$ ), preterm birth (OR $1.41,95 \% \mathrm{Cl} 1.33$ to $1.50, \mathrm{I}^{2} 70.97 \%$ ) and low birth weight (OR $1.40,95 \% \mathrm{Cl} 1.19$ to $1.61, \mathrm{I}^{2} 99.85 \%$ ). Limitations relate to available data, unmeasured confounders and the small number of studies for some outcomes.

Conclusions This review identified consistent evidence that lower occupational status, especially manual occupations and unemployment, were significantly associated with increased risk of multiple adverse pregnancy outcomes. Strategies to improve pregnancy outcomes should incorporate approaches that address wider determinants of health to provide women and families with the best chances of having a healthy pregnancy and baby and to decrease pregnancy-related health inequalities in the general population.

\section{Strengths and limitations of this study}

- Search strategy broad and wide-ranging resulting in the inclusion of 17130631 mothers and babies in the analysis from 35 studies.

- No restriction by pregnancy outcome; results therefore provide a comprehensive overview of the effects of socioeconomic status.

- Results are limited to the UK and Ireland only.

- Between countries and over time, the occupational classifications used have changed.

- Occupational data dominates the evidence base; limited data were available for other measures of inequality (eg, housing, income and car ownership).

PROSPERO registration number PROSPERO CRD42019140893.

\section{INTRODUCTION}

There has been a sustained increase in infant mortality in England, disproportionately affecting the most deprived areas, ${ }^{12}$ and a reduction in life expectancy for the first time in 120 years, with similar trends in the rest of the UK. ${ }^{1}$ The evidence base that demonstrates the increase in children and families living in poverty, and the association with increasing mortality rates and reduction in life expectancy, is compelling. The recent Marmot review ${ }^{1}$ identified that $30 \%$ of children in England are living in poverty, and those in the most deprived $10 \%$ are almost twice as likely to die and more likely to have illness or long-term disability.

Having a healthy pregnancy is vital to improve the life chances of future generations, to prevent perinatal and infant mortality and to give every child the best start in life. For example, babies born small for gestational age (SGA) or preterm are more likely 
to die during infancy and childhood. ${ }^{3-5}$ However, studies in high-income countries (HICs) show worse pregnancy outcomes among women living in deprived communities. $^{67}$ Socioeconomic inequalities disproportionately affect women, which impacts on their own health and the life chances of their children. Female life expectancy has declined in England's most deprived areas over the past 10 years, which has been attributed to austerity, whereas it has slightly increased for men. ${ }^{1}$ Women, particularly lone mothers, have the highest gap between income and adequate living standards and are the most likely to be living in food insecure households, ${ }^{8}$ which is another measure of poverty. The evidence relating to the disproportionate impact of the COVID-19 pandemic on the most disadvantaged, and the potential for future inequalities in health consequences due to political and economic pathways, ${ }^{9}$ will only serve to increase these inequalities.

Compared with other HICs, the UK and Republic of Ireland (ROI) perform poorly in terms of both infant and maternal mortality. ${ }^{2} 1011$ For example, differences in infant mortality are linked to the level of deprivation, and there is evidence for widening inequalities in the poorest households since 2013. ${ }^{2}$ Slowdown in life expectancy has been worse in the UK than almost all other HICs across Europe, whereas wealth inequality has increased faster. ${ }^{1}$ Health costs associated with inequalities in England are estimated to be more than $£ 12$ billion $^{1}$; therefore, there are potential economic benefits as well as moral and ethical reasons to invest in reducing health inequalities. Despite this, local governments responsible for delivering public health agendas in England have had a 77\% budget reduction over 10 years. These cuts have been inequitable, regressive and applied to the most deprived areas of England with the greatest health needs, contributing to widening health inequalities. ${ }^{1}$

Central to understanding the relatively low performance of the UK and ROI compared with other HICs is documenting the scale of inequalities related to adverse pregnancy outcomes across the social gradient. Important individual factors to explore the 'causes of the causes $^{1}$ include employment, education, income and housing. While there have been a number of systematic reviews that examine the impact of deprivation, ${ }^{6} 1213$ these are out of date and focus on area-based measures (such as the Index of Multiple Deprivation) as an inadequate proxy for individual measures of socioeconomic status (SES), which can be more difficult to collate. There is some evidence from a meta-analysis published in $2012^{14}$ that individual-level social class is significantly associated with an increase in low birth weight (LBW) and infant mortality, and the authors concluded that future research should focus on the more proximal individual-level determinants of health. Using individuallevel socioeconomic indicators provides a richer way of understanding the relative importance of particular SES factors and their direct and indirect impact on a range of adverse pregnancy outcomes. This is an essential first step to understand and then mitigate their effect on the most vulnerable groups in our communities. This systematic review and meta-analysis aimed to understand the association between individual-level and household-level measures of SES and adverse pregnancy outcomes in the UK and ROI. We hypothesised that there would be an association between low SES measures and increased risk of adverse pregnancy outcomes.

\section{METHODS}

\section{Protocol, search strategy and study selection}

The systematic review was registered with PROSPERO (CRD42019140893) and conducted in line with MOOSE guidelines $^{15}$ (online supplemental appendix S1). Nine electronic databases were searched between 1 January 1999 and 7 August 2019 (online supplemental appendix S2). The start date of 1999 was included as it coincided with the start of the English Health Inequalities Strategy. ${ }^{16}$ The databases were searched using key words and Medical Subject Headings (MeSH) developed by an information specialist at Newcastle University (CR).

Searching for epidemiological studies using databases alone systematically misses a proportion of relevant studies; therefore, in line with MOOSE guidelines, supplementary searches were carried out. Backwards and forwards citation chaining of included studies were undertaken and relevant systematic reviews were assessed. Finally, literature searches comprising key data sources for the UK and ROI (online supplemental appendix S3) were run, and experts in key organisations in each of the countries were contacted. ${ }^{17}$

The inclusion criteria for the review were determined a priori in terms of Population, Exposure, Comparison, Outcome and Study design ${ }^{18}$ :

- Population: the unborn fetus, neonate (child under 28 days of age) or mother in the UK or ROI.

- Exposure: any individual-level or household-level socioeconomic factor such as employment, education, income, housing, poverty/low wage, social isolation and car ownership. Aggregate area-level measures of SES (such as the Index of Multiple Deprivation) were excluded.

- Comparison: studies with individual-level or household-level exposures that compare low to high SES.

- Outcomes: pregnancy outcomes related to the fetus, neonate or mother. We did not exclude outcomes if they were relevant to the fetus, neonate or mother during pregnancy or within 28 days postnatal as we wanted to provide a comprehensive overview of the existing evidence base.

- Study design: observational studies with cohort, casecontrol, longitudinal or cross-sectional designs.

The results from the database searches were imported into Endnote, ${ }^{19}$ and duplicates were removed. Screening the titles and abstracts was managed in Covidence. ${ }^{20}$ Title, abstract and full-text screening were completed independently in duplicate. Where multiple studies reported 
data from the same cohort, decisions on which study to include were based on sample size, time period and categories used to define determinants and outcomes. We included any studies that met the inclusion criteria and reported an association between the exposure and outcomes of interest, regardless of whether this was the primary aim of the study. Decisions on inclusion and exclusion of studies were recorded using a Preferred Reporting Items for Systematic Reviews and Meta-Analyses flow chart.

\section{Data extraction and quality appraisal}

Data extractions were carried out using a standardised protocol independently by two reviewers for each included study (KT, OA and MM), and discrepancies were validated by a third reviewer $(\mathrm{AO}$ and $\mathrm{NH})$. Data were extracted on the characteristics of the included studies and the results reported on associations between inequality exposure variables and pregnancy outcomes (online supplemental appendix S4). Authors of primary studies were contacted if needed to request additional data or clarify reported results (online supplemental appendix S5). The Newcastle-Ottawa scale was used to assess information bias, selection bias and confounding in case-control and cohort studies (online supplemental appendix S6).${ }^{21}$ Stars were awarded for high-quality aspects of study design. A maximum of eight stars was attainable for cohort studies (0-2 stars deemed as low quality, 3-5 moderate quality and 6-8 high quality) and nine stars for case-control studies (0-3 stars for low quality, 4-6 for moderate quality and 7-9 for high quality). Any discrepancies on screening, extraction and quality appraisal were resolved through discussion between the reviewers (KT, $\mathrm{OA}, \mathrm{MM}$ and $\mathrm{AO}$ ) and the project lead $(\mathrm{NH})$.

The Grading of Recommendations, Assessment, Development and Evaluation (GRADE) methodology was used to address the quality of the evidence. ${ }^{22}$ Only those outcomes considered most important were evaluated using GRADE, and due to the available evidence, only inequalities in occupation/social class were considered. Quality of evidence for each pregnancy outcome was assessed based on study design (the baseline rating for observational studies is low), risk of bias, imprecision of estimates, inconsistency of results from different studies, indirectness of study results (ie, lack of applicability) and publication bias. ${ }^{23}$

\section{Evidence synthesis and statistical analysis}

A meta-analysis was used to calculate a pooled odds ratio (OR) and a 95\% CI where there were at least two studies reporting the same outcome and comparable individuallevel or household-level SES exposures. Meta-analysis was only possible for occupation-derived social class. Subgroups for this variable were included in the metaanalysis where possible to stratify analysis comparing intermediate-level or lowest-level of occupation/social class to highest level. Heterogeneity was explored through meta-regression for factors including duration of data collected, country, region, date and sample size, location, publication date and quality. $\mathrm{I}^{2}$ values of $25 \%$, $50 \%$ and $75 \%$ were used to indicate low, moderate and high levels of heterogeneity. ${ }^{24}$ Publication bias was investigated using Egger's test and funnel plots. Sensitivity analysis was performed for each meta-analysis by excluding one dataset at a time to identify the effect of any individual dataset on the pooled effect size and between-study heterogeneity. All analyses were conducted in Stata V.16. ${ }^{25}$

Narrative synthesis methods described by Popay et $a t^{26}$ were used to report outcomes with insufficient data to include in a meta-analysis and to integrate the narrative and meta-analysis data. Preliminary synthesis involved grouping all data into themes based on outcomes reported and type of SES measure. The associations between SES determinants and pregnancy outcomes reported by the studies, or calculated using data reported in the studies, were tabulated according to pregnancy outcome themes (eg, birth weight outcomes) and subgrouped by the level of SES determinant (eg, occupation). Where required, data were transformed to be comparable between studies (eg, using reported frequency data to recalculate ORs into more comparable SES categories). Authors were contacted when insufficient data were reported in the published studies to calculate ORs. Relationships between studies are reported by discussion of reciprocal and refutational patterns in the data, including the statistical significance and direction of effect. The narrative synthesis is supplemented by a discussion of the wider evidence base and the strengths and limitations of this review including the gaps in the existing literature.

\section{Patient and public involvement}

Patients or the public were not involved in any aspect of the study design, conduct or in the development of the research question or outcome measures.

\section{RESULTS}

The database searches identified 53821 records after duplicates had been removed. A further 9414 references and citations and 529 grey literature data sources were screened (figure 1), of which 1439 were screened at fulltext level (reasons for exclusion are detailed in online supplemental appendix S7). Forty-one studies met the inclusion criteria, six of these reported duplicate data (online supplemental appendix S8). Thirty-five studies were included reporting unique data for $17 \quad 130631$ mothers and babies, 27 were conducted in the UK and 8 in the ROI (table 1). The studies were published between the years 1999 and 2019 (with data reported from 1990 onwards), 10 were case control and 25 were cohort studies. The majority of studies reported national-level data, and these datasets are assumed to be nationally representative. All studies scored between three and eight on the Newcastle-Ottawa Scale (online supplemental appendix S9). The studies were rated as either high quality $(n=4$ case-control, $\mathrm{n}=18$ cohort) or moderate quality $(\mathrm{n}=6$ 


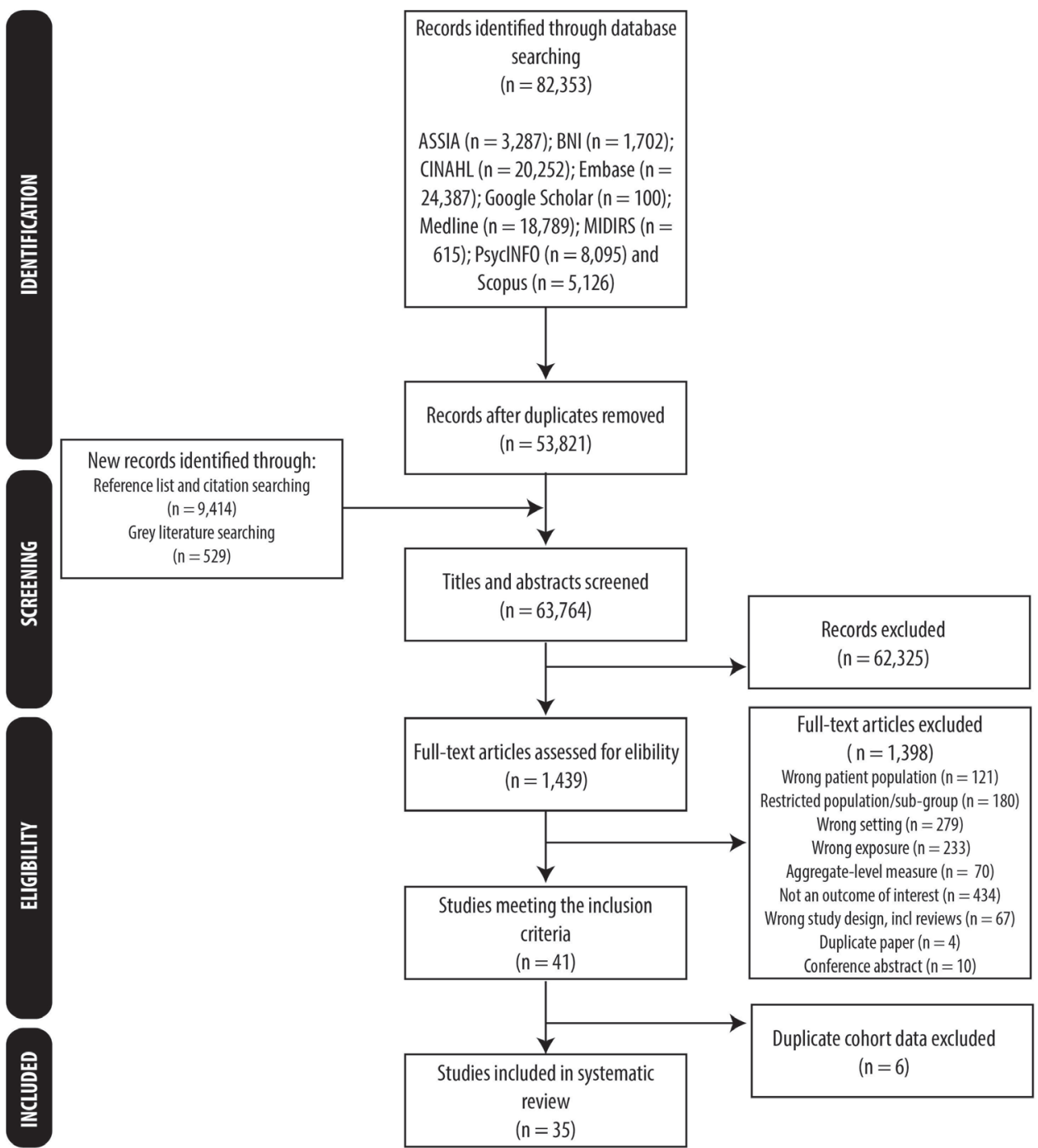

Figure 1 PRISMA flow chart. PRISMA, Preferred Reporting Items for Systematic Reviews and Meta-Analyses.

case-control, $\mathrm{n}=7$ cohort), with no low-quality studies. Most studies $(\mathrm{n}=23)$ reported outcomes associated with occupation-derived social class using different classifications (online supplemental appendix S10), which were grouped into: (1) highest level (managerial/professional) occupations; (2) intermediate-level occupations; and (3) lowest level (routine/manual/partly skilled) occupations. ${ }^{27}$ Seventeen studies investigated associations with education, seven for employment and income/ finances, four for housing and one for car ownership. Pregnancy outcomes reported were mortality (stillbirth, neonatal, perinatal and maternal), LBW, preterm birth (PTB), mode of delivery, maternal morbidity and congenital anomalies (table 1).

\section{Mortality}

Six studies reported stillbirth, neonatal mortality, perinatal mortality and maternal mortality (online supplemental appendix S11 tables a-d). Individual and household inequality measures reported were paternal, maternal and primary household occupation/social class.
Stillbirth was reported by five studies ${ }^{28-32}$ for 12642203 births (online supplemental appendix S11, table a), and four ${ }^{29-32}$ could be pooled into the meta-analysis. Perinatal mortality was reported by three studies ${ }^{293132}$ for 11189810 births (online supplemental appendix S11, table b), and neonatal mortality was reported by three studies ${ }^{30-32}$ for 11580610 births (online supplemental appendix S11, table c), and all could be pooled in meta-analysis. There were significantly increased odds for the pooled lowest/ intermediate occupation compared with highest for all three outcomes (stillbirth: OR $1.4095 \%$ CI 1.23 to 1.59 (figure 2); perinatal mortality: OR 1.39 95\% CI 1.23 to 1.57 (figure 3); neonatal mortality: OR 1.39 95\% CI 1.22 to 1.57 (figure 4)). All subgroup meta-analyses showed highest odds among the lowest level of occupation/ social class compared with the highest level (stillbirth: OR $1.6195 \%$ CI 1.37 to 1.88 ; perinatal mortality: OR $1.5795 \%$ CI 1.41 to 1.74 ; neonatal mortality: OR 1.58 , $95 \%$ CI 1.44 to 1.74; figures 2-4). Heterogeneity was high for all meta-analyses and was not explained by any of the factors included in meta-regression (online 
Table 1 Summary of the included studies

\begin{tabular}{lll}
$\begin{array}{l}\text { Author, } \\
\text { publication year, } \\
\text { country }\end{array}$ & $\begin{array}{l}\text { Study design and } \\
\text { period }\end{array}$ \\
\hline $\begin{array}{l}\text { Brick et al 2016, } \\
\mathrm{ROI}^{95}\end{array}$ & Cohort study 2009 \\
& & \\
& & \\
Bush et al 2013, & Case-control study \\
UK &
\end{tabular}

Registry/data source

National Perinatal Reporting System (NPRS) and the Hospital In-Patient Enquiry scheme (HIPE).

The UK Obstetric

Surveillance System (UKOSS)
Description of population, sample size

All nulliparous singleton births to women discharged from the 19 publicly funded hospital maternity units for whom an NPRS and HIPE record was available. $n=29870$.

Cases were from any consultant-led

midwifery unit in the UK reporting

Occupation/ Myocardial infarction. 5 myocardial infarction. The controls were the two women who delivered immediately before the cases.

Cases: $n=25$.

Controls: $n=1360$.

$\begin{array}{lll}\begin{array}{l}\text { Clemens and } \\ \text { Dibben 2016, } \\ \text { Scotland }\end{array} & \begin{array}{l}\text { Cohort study 1994- } \\ 2008\end{array} & \begin{array}{l}\text { The Scottish } \\ \text { Longitudinal Study } \\ \text { (SLS) and the } \\ \text { Scottish Morbidity } \\ \text { Record (SMR). }\end{array} \\ \begin{array}{l}\text { Collingwood } \\ \begin{array}{l}\text { Bakeo and Clarke } \\ \text { 2006, England and } \\ \text { Wales }\end{array}\end{array} & \begin{array}{l}\text { Cohort study 1990s } \\ \text { Office for National }\end{array} & \begin{array}{l}\text { Statistics (ONS) } \\ \text { Longitudinal Study. }\end{array}\end{array}$

Singleton births from the SLS, a $5 \%$ sample of the Scottish population that links census records and maternity hospital inpatient data from the SMR. Sample size not reported.

Singleton, live births to mothers in the ONS Longitudinal Study, a 1\% sample of the population of England and Wales for which linked census data are available. $\mathrm{n}=57129$ (no. of rooms/cars), $\mathrm{n}=49212$ (economic activity) and $n=56988$ (no. of people in household)

$\begin{array}{lll}\begin{array}{l}\text { Dibben et al 2006, Cohort study 1996- } \\ \text { England }^{88}\end{array} & \begin{array}{l}\text { Births and deaths } \\ \text { recorded by local } \\ \text { registrars and } \\ \text { forwarded to the } \\ \text { ONS. }\end{array} \\ \begin{array}{lll}\text { Essex et al 2013, } \\ \text { UK }\end{array} & \begin{array}{l}\text { Cohort study 2000- } \\ 2002\end{array} & \begin{array}{l}\text { Millennium Cohort } \\ \text { Study (MCS). }\end{array}\end{array}$

Births registered in England and Wales by local registrars. Parents' age obtained from the ONS and birth weight derived from birth notifications supplied to local registrars by the National Health Service. $\mathrm{n}=306067$

Live singleton births whose parents were enrolled in the MCS. The MCS is a retrospective study designed to disproportionately include comparative samples of children born in advantaged, disadvantaged and ethnically diverse regions of England, Scotland, Northern Ireland and Wales. Eligible births were selected from a random sample of UK electoral wards using the register of children eligible for child benefit. Data were collated using interviews and linked in turn to hospital medical records. $\mathrm{n}=18239$.

\begin{tabular}{|c|c|c|c|c|c|c|}
\hline $\begin{array}{l}\text { Fairley and } \\
\text { Leyland } 2006 \text {, } \\
\text { Scotland }^{79}\end{array}$ & $\begin{array}{l}\text { Cohort study 1980- } \\
2000\end{array}$ & $\begin{array}{l}\text { Data linked to the } \\
\text { registrar general's } \\
\text { birth registration. }\end{array}$ & $\begin{array}{l}\text { All live singleton hospital births in } \\
\text { Scotland linked to registrar general's birth } \\
\text { registrations. } \\
n=283032(1990-1994) \text { and } \\
n=281001(1995-2000) \text {. }\end{array}$ & $\begin{array}{l}\text { Occupation/ } \\
\text { social class. }\end{array}$ & $\begin{array}{l}\text { Low birth weight. } \\
\text { Preterm birth. } \\
\text { Small for gestational } \\
\text { age. }\end{array}$ & $\begin{array}{l}5 \\
\text { Moderate }\end{array}$ \\
\hline $\begin{array}{l}\text { Fairley et al 2011, } \\
\text { Scotland }^{97}\end{array}$ & $\begin{array}{l}\text { Cohort study 1990- } \\
\text { 1991, 1999-2000. }\end{array}$ & $\begin{array}{l}\text { Information Services, } \\
\text { National Health } \\
\text { Service National } \\
\text { Services Scotland. }\end{array}$ & $\begin{array}{l}\text { Routine maternity discharge data from live } \\
\text { singleton births in Scottish hospitals from } \\
\text { two time periods. } n=105247 \text { (1990) and } \\
n=70667 \text { (1999-2000). }\end{array}$ & $\begin{array}{l}\text { Occupation/ } \\
\text { social class. }\end{array}$ & Mode of delivery. & $\begin{array}{l}7 \\
\text { High }\end{array}$ \\
\hline $\begin{array}{l}\text { Fitzpatrick et al } \\
2012, \text { UK }^{100}\end{array}$ & $\begin{array}{l}\text { Case-control study } \\
2010-2011 .\end{array}$ & UKOSS. & $\begin{array}{l}\text { Cases were from any obstetrician-led } \\
\text { maternity unit in the UK reporting cases } \\
\text { of placenta accrete/increta/percreta. } \\
\text { The controls were the two women who } \\
\text { delivered immediately before the case in } \\
\text { the same hospital. } \\
\text { Cases: } n=134 \text {. } \\
\text { Controls: } n=256 \text {. }\end{array}$ & $\begin{array}{l}\text { Occupation/ } \\
\text { social class. }\end{array}$ & $\begin{array}{l}\text { Placenta accreta/ } \\
\text { increta/percreta. }\end{array}$ & $\begin{array}{l}5 \\
\text { Moderate }\end{array}$ \\
\hline
\end{tabular}

Continued

exposure Pregnancy outcome Risk of bias

Occupation/ Mode of delivery. 6 social class. High social class. Moderate

$\begin{array}{ll}\begin{array}{l}\text { Education. } \\ \text { Occupation/ } \\ \text { social class. }\end{array} & \text { Preterm birth. } \\ & \text { Moderate } \\ \begin{array}{l}\text { Employment. Low birth weight. } \\ \begin{array}{l}\text { Housing. } \\ \text { Car }\end{array}\end{array} & \begin{array}{l}7 \\ \text { High }\end{array}\end{array}$
ownership.

$\begin{array}{lll}\text { Income. }^{1} \quad \text { Low birth weight. } & \begin{array}{l}7 \\ \text { High }\end{array} \\ & & \\ & & \\ \begin{array}{l}\text { Education. } \\ \text { Occupation/ } \\ \text { social class. }\end{array} & \text { Mode of delivery. } & 5 \\ \end{array}$

social class.

Inequality 
Table 1 Continued

Author,

publication year, Study design and

country $\begin{array}{ll}\text { Fitzpatrick et al } & \text { Case-control study } \\ 2015, \text { UK }^{105} & \text { 2005-2014 }\end{array}$

\section{Registry/data}

source Description of population, sample size

Cases were from any obstetrician-led maternity unit in the UK reporting cases of amniotic-fluid embolism (AFE) (or a severe maternal outcome caused by AFE). The controls were the two women who delivered immediately before the case in the same hospital.

Cases: $n=120$.

Controls: $n=3834$.

\begin{tabular}{|c|c|c|c|}
\hline $\begin{array}{l}\text { Gardosi et al 2013, } \\
\text { England }^{28}\end{array}$ & $\begin{array}{l}\text { Cohort study 2009- } \\
2011\end{array}$ & $\begin{array}{l}\text { Perinatal episode } \\
\text { electronic record } \\
\text { (PEER). }\end{array}$ & $\begin{array}{l}\text { Births within the } 19 \text { maternity units in the } \\
\text { West Midlands using the database derived } \\
\text { from the regional NHSnet-based PEER } \\
\text { hosted by the West Midlands Perinatal } \\
\text { Institute. } n=105476 \text {. }\end{array}$ \\
\hline $\begin{array}{l}\text { Knight et al 2008, } \\
\text { UK }^{130}\end{array}$ & $\begin{array}{l}\text { Case-control study } \\
2005-2006\end{array}$ & UKOSS. & $\begin{array}{l}\text { Cases were from any consultant- } \\
\text { led obstetric unit in the UK reporting } \\
\text { pulmonary embolism (confirmed using } \\
\text { imaging, surgery/postmortem or clinician } \\
\text { diagnosis). The controls were the two } \\
\text { women who delivered immediately before } \\
\text { the case in the same hospital. } \\
\text { Cases: } n=141 \text {. } \\
\text { Controls: } n=259 \text {. }\end{array}$ \\
\hline
\end{tabular}

$\begin{array}{lll}\begin{array}{l}\text { Martinson and } \\ \text { Reichman 2016, } \\ \text { UK }\end{array}{ }^{83} & \begin{array}{l}\text { Cohort study 2000- } \\ 2002\end{array} & \begin{array}{l}\text { MCS and The UK } \\ \text { data archive. }\end{array} \\ \begin{array}{l}\text { Matijasevich et al } \\ \text { 2012, England }\end{array} & \begin{array}{l}\text { Cohort study 1991- } \\ 1992\end{array} & \begin{array}{l}\text { The Avon } \\ \text { Longitudinal Study of } \\ \text { Parents and Children } \\ \text { (ALSPAC). }\end{array}\end{array}$

$\begin{array}{lll}\begin{array}{l}\text { McAvoyet al 2006, Cohort study 1999- } \\ \mathrm{ROI}^{80}\end{array} & \begin{array}{l}\text { Eastern Regional } \\ \text { Health Authority } \\ \text { (ERHA). }\end{array} \\ \begin{array}{ll}\text { Murrin et al 2007, } \\ \mathrm{ROI}^{85}\end{array} & \begin{array}{l}\text { Cohort study 2001- } \\ 2003\end{array} & \text { Lifeways cohort. }^{3}\end{array}$

singleton births whose parents were enrolled in the MCS. Data were collated from an interview conducted by a home visitor within 9 months of delivery. $\mathrm{n}=12018$.

Women with a singleton, liveborn

and a term pregnancy mode of

longitudinal cohort study in Avon, England. $\mathrm{n}=13678$.

All births in the ERHA (encompassing the Northern, the East Coast and the South Western Area health boards). $\mathrm{n}=63571$.

Women involved in the Lifeways cohort who were Irish-born and recruited in either a predominantly rural region (University College Hospital Galway) or an urban area (Coombe Women's Hospital in Dublin). Baseline data for mothers were collected using a self-completed questionnaire. Pregnancy outcomes were derived from hospital records. $n=1048$.

\begin{tabular}{|c|c|c|c|c|c|c|}
\hline $\begin{array}{l}\text { Nair et al 2014, } \\
\text { UK }^{101}\end{array}$ & $\begin{array}{l}\text { Case-control study } \\
2005-2013\end{array}$ & UKOSS. & $\begin{array}{l}\text { Cases were from any consultant-led } \\
\text { obstetric unit in the UK reporting severe } \\
\text { maternal morbidity. The controls were } \\
\text { women who delivered immediately before } \\
\text { the cases in the same hospital. } \\
\text { Cases: } n=1753 \text {. } \\
\text { Controls: } n=3310 \text {. }\end{array}$ & $\begin{array}{l}\text { Occupation/ } \\
\text { social class. }\end{array}$ & Maternal morbidity. & $\begin{array}{l}7 \\
\text { High }\end{array}$ \\
\hline $\begin{array}{l}\text { Nair et al 2016, } \\
\text { UK }^{33}\end{array}$ & $\begin{array}{l}\text { Case-control study } \\
2009-2013\end{array}$ & $\begin{array}{l}\text { Mothers and Babies: } \\
\text { Reducing Risk } \\
\text { through Audits and } \\
\text { Confidential Enquiries } \\
\text { across the UK } \\
\text { (MBRRACE-UK) and } \\
\text { UKOSS. }\end{array}$ & $\begin{array}{l}\text { Cases were from women who died from } \\
\text { direct or indirect causes during pregnancy } \\
\text { or within } 42 \text { days of the end of pregnancy } \\
\text { in the UK. Population controls were } \\
\text { obtained from UKOSS. } \\
\text { Cases: } n=383 \text {. } \\
\text { Controls: } n=3310 \text {. }\end{array}$ & Employment. & Maternal mortality. & $\begin{array}{l}7 \\
\text { High }\end{array}$ \\
\hline $\begin{array}{l}\text { National Perinatal } \\
\text { Reporting System } \\
\text { (NPRS), 1990- } \\
1993 \text { and 2002- } \\
2018, \text { ROI }^{4} 29\end{array}$ & $\begin{array}{l}\text { Cohort study 1990- } \\
\text { 1993; 1999-2016 }\end{array}$ & NPRS. & $\begin{array}{l}\text { The NPRS collects information on birth } \\
\text { records each year from } 19 \text { maternity } \\
\text { units and all practising self-employed } \\
\text { community midwives. } \\
n=1352878 \text {. }\end{array}$ & $\begin{array}{l}\text { Occupation/ } \\
\text { social class. }\end{array}$ & $\begin{array}{l}\text { Very low birth weight } \\
\text { (VLBW). } \\
\text { Low birth weight. } \\
\text { Mid-low birth weight. } \\
\text { Macrosomia. } \\
\text { Stillbirth. } \\
\text { Neonatal death. } \\
\text { Perinatal death. }\end{array}$ & $\begin{array}{l}6 \\
\text { High }\end{array}$ \\
\hline
\end{tabular}

Continued

\section{Inequality}

exposure Pregnancy outcome Risk of bias

Occupation/ Amniotic-fluid 7

social class. embolism (AFE). High

Severe maternal

outcome caused by

AFE.

Occupation/ Pulmonary embolism. 7

social class. High

Education. Low birth weight. 4

Moderate

Education. Mode of delivery. 7

Income. Preterm birth. High

Intrauterine growth

restriction.

Employment. Low birth weight.

Occupation/ Moderate

social class.

Education. Mid-low birth weight. 6

Macrosomia. High

High


Table 1 Continued

\begin{tabular}{lll}
\hline $\begin{array}{l}\text { Author, } \\
\text { publication year, } \\
\text { country }\end{array}$ & $\begin{array}{l}\text { Study design and } \\
\text { period }\end{array}$ & $\begin{array}{l}\text { Registry/data } \\
\text { source }\end{array}$ \\
\hline $\begin{array}{l}\text { National Records } \\
\text { of Scotland (NRS), }\end{array}$ & $\begin{array}{l}\text { Cohort study 2000 - } \\
2019^{30}\end{array}$ & NRS. \\
& & \\
\hline $\begin{array}{l}\text { Niedhammer et al } \\
\text { 2009, ROI }\end{array}$ & $\begin{array}{l}\text { Cohort study 2001- } \\
2003\end{array}$ & Lifeways cohort. \\
& & \\
& & \\
Niedhammer et al & $\begin{array}{l}\text { Cohort study 2001- } \\
\text { 2012, ROI }\end{array}$ & Lifeways cohort.
\end{tabular}

Description of population, sample size exposure

Inequality

Pregnancy outcome Risk of bias

Births registered in Scotland by local registrars. Birth records derived from Occupation/ Stillbirths. 6 notifications supplied to local registrars by the National Health Service. $n=1675973$.

social class. Perinatal mortality. High

Neonatal mortality.

Women involved in the Lifeways cohort Education. ${ }^{5} \quad$ Low birth weight. 7

who were Irish born and recruited in either Occupation/ Mid-low birth weight. High a predominantly rural region or an urban social class. Preterm birth. ${ }^{5}$ area.

$n=1124$.

Small for gestational age.

Women involved in the Lifeways cohort Education. Preterm birth. 7 who were Irish-born and recruited in either Housing. High a predominantly rural region or an urban Income. area. $n=924$.

\begin{tabular}{|c|c|c|c|c|c|c|}
\hline $\begin{array}{l}\text { ONS 1999-2017, } \\
\text { England and } \\
\text { Wales }^{31}\end{array}$ & $\begin{array}{l}\text { Cohort study 1999- } \\
2017\end{array}$ & ONS. & $\begin{array}{l}\text { Births registered in England and Wales by } \\
\text { local registrars. Birth records derived from } \\
\text { notifications supplied to local registrars by } \\
\text { the National Health Service. } \\
n=12799233 \text {. }\end{array}$ & $\begin{array}{l}\text { Occupation/ } \\
\text { social class. }\end{array}$ & $\begin{array}{l}\text { VLBW. } \\
\text { Low birth weight. } \\
\text { Mid-low birth weight. } \\
\text { Macrosomia. } \\
\text { Stillbirths. } \\
\text { Perinatal mortality. } \\
\text { Neonatal mortality. }\end{array}$ & $\begin{array}{l}6 \\
\text { High }\end{array}$ \\
\hline $\begin{array}{l}\text { Patel et al 2005, } \\
\text { England }^{98}\end{array}$ & $\begin{array}{l}\text { Cohort study 1990- } \\
1991\end{array}$ & $\begin{array}{l}\text { Avon Longitudinal } \\
\text { Study of Parents and } \\
\text { Children (ALSPAC). }\end{array}$ & $\begin{array}{l}\text { Women with a singleton, liveborn } \\
\text { infant and a term pregnancy mode of } \\
\text { delivery who were involved in ALSPAC, a } \\
\text { longitudinal cohort study in Avon, England. } \\
\mathrm{n}=8925 \text { (maternal social class). } \\
\mathrm{n}=12078 \text { (home ownership). }\end{array}$ & $\begin{array}{l}\text { Occupation/ } \\
\text { social class. } \\
\text { Employment. } \\
\text { Housing. }\end{array}$ & Mode of delivery. & $\begin{array}{l}5 \\
\text { Moderate }\end{array}$ \\
\hline
\end{tabular}

$\begin{array}{lll}\begin{array}{l}\text { Poulsen et al } \\ \text { 2015, England }\end{array} & \begin{array}{l}\text { Cohort study 2007- } \\ 2010\end{array} & \begin{array}{l}\text { The Born in Bradford } \\ (\mathrm{BiB}) \text { cohort. }\end{array} \\ & \\ \text { Scott et al 2012, } & \begin{array}{l}\text { Case-control study } \\ \text { 2007-2010 }\end{array} & \text { UKOSS. } \\ \text { UK }^{104} & & \end{array}$

Women with singleton live births who consented to take part in the BiB study (who attended the antenatal service in Bradford and were booked to give birth in Bradford). A baseline questionnaire was used together with data recorded in the electronic maternity system.

$\mathrm{n}=10850$.

Cases were from any consultant-led obstetric unit in the UK reporting any pregnant women who had an antenatal Education. Preterm birth. 5 stroke (confirmed with imaging or at postmortem). The control patients were the women who delivered immediately before the case in the same hospital.

Cases: $n=30$.

Controls: $\mathrm{n}=89$.

\begin{tabular}{|c|c|c|c|c|c|c|}
\hline $\begin{array}{l}\text { Sheridan et al } \\
\text { 2013, England }\end{array}$ & $\begin{array}{l}\text { Cohort study 2007- } \\
2011\end{array}$ & $\begin{array}{l}\text { BiB and British } \\
\text { Isles Network of } \\
\text { Congenital Anomalies } \\
\text { Register. }\end{array}$ & $\begin{array}{l}\text { Women with singleton live births who } \\
\text { consented to take part in the BiB study. } \\
\text { Anomalies were classified into six groups: } \\
\text { single anomalies, several anomalies within } \\
\text { the same anomaly group, metabolic } \\
\text { disorders, syndromal associations, chromo } \\
\text { somal syndromes and more than one } \\
\text { unrelated anomaly. } \\
n=13776 \text {. }\end{array}$ & Education. & $\begin{array}{l}\text { Congenital } \\
\text { anomalies. }\end{array}$ & $\begin{array}{l}6 \\
\text { High }\end{array}$ \\
\hline $\begin{array}{l}\text { Sinnott et al 2016, } \\
\text { ROI }^{99}\end{array}$ & Cohort study 2009 & $\begin{array}{l}\text { NPRS and HIPE } \\
\text { scheme. }\end{array}$ & $\begin{array}{l}\text { All women with singleton births (live and } \\
\text { stillborn) discharged from } 19 \text { publicly } \\
\text { funded hospitals for whom NPRS and } \\
\text { HIPE data were available. } \\
n=69304 \text {. }\end{array}$ & $\begin{array}{l}\text { Occupation/ } \\
\text { social class. }\end{array}$ & Induction of labour. & $\begin{array}{l}6 \\
\text { High }\end{array}$ \\
\hline $\begin{array}{l}\text { Snelgrove and } \\
\text { Murphy 2015, } \\
\text { UK }^{93}\end{array}$ & $\begin{array}{l}\text { Cohort study 2000- } \\
2002\end{array}$ & $\begin{array}{l}\text { MCS and The UK } \\
\text { data archive. }\end{array}$ & $\begin{array}{l}\text { Live singleton births whose parents } \\
\text { were enrolled in the MCS. Data were } \\
\text { collated from an interview conducted by } \\
\text { a home visitor within } 9 \text { months of delivery. } \\
n=17285 \text {. }\end{array}$ & $\begin{array}{l}\text { Education. } \\
\text { Employment. } \\
\text { Housing. } \\
\text { Income. } \\
\text { Occupation/ } \\
\text { social class. }\end{array}$ & Preterm birth. & $\begin{array}{l}7 \\
\text { High }\end{array}$ \\
\hline
\end{tabular}

Continued 
Table 1 Continued

\begin{tabular}{|c|c|c|c|c|c|c|}
\hline $\begin{array}{l}\text { Author, } \\
\text { publication year, } \\
\text { country }\end{array}$ & $\begin{array}{l}\text { Study design and } \\
\text { period }\end{array}$ & $\begin{array}{l}\text { Registry/data } \\
\text { source }\end{array}$ & Description of population, sample size & $\begin{array}{l}\text { Inequality } \\
\text { exposure }\end{array}$ & Pregnancy outcome & Risk of bias \\
\hline $\begin{array}{l}\text { Stacey et al 2016, } \\
\text { England }^{86}\end{array}$ & $\begin{array}{l}\text { Cohort study 2007- } \\
2010\end{array}$ & BiB. & $\begin{array}{l}\text { Women with singleton live births who } \\
\text { consented to take part in the BiB study. A } \\
\text { baseline questionnaire was used together } \\
\text { with data recorded in the electronic } \\
\text { maternity system. }\end{array}$ & $\begin{array}{l}\text { Education. }^{7} \\
\text { Occupation/ } \\
\text { social class. } \\
\text { Income/ } \\
\text { finances. }\end{array}$ & $\begin{array}{l}\text { Preterm birth. }{ }^{7} \\
\text { Small for gestational } \\
\text { age. }\end{array}$ & $\begin{array}{l}8 \\
\text { High }\end{array}$ \\
\hline
\end{tabular}

\begin{tabular}{|c|c|c|c|c|c|c|}
\hline $\begin{array}{l}\text { Tuthill et al 1999, } \\
\text { Wales }^{32}\end{array}$ & $\begin{array}{l}\text { Cohort study 1993- } \\
1995\end{array}$ & $\begin{array}{l}\text { All Wales Perinatal } \\
\text { Survey/ Child Health } \\
\text { System Database/ } \\
\text { ONS/ Cardiff Births } \\
\text { Survey. }\end{array}$ & $\begin{array}{l}\text { Data relating to stillbirths and infant } \\
\text { deaths were identified using the All Wales } \\
\text { Perinatal Survey. Survivors born to women } \\
\text { resident in South Glamorgan (Wales) } \\
\text { recorded prospectively by the Cardiff } \\
\text { Births Survey were used as a comparison. } \\
n=16842 \text {. }\end{array}$ & $\begin{array}{l}\text { Occupation/ } \\
\text { social class. }\end{array}$ & $\begin{array}{l}\text { Stillbirth. } \\
\text { Neonatal death. } \\
\text { Perinatal death. }\end{array}$ & $\begin{array}{l}4 \\
\text { Moderate }\end{array}$ \\
\hline $\begin{array}{l}\text { Vinturache et al } \\
2017, \mathrm{ROI}^{94}\end{array}$ & $\begin{array}{l}\text { Cohort study 2009- } \\
2013\end{array}$ & $\begin{array}{l}\text { Coombe Women and } \\
\text { Infants University } \\
\text { Hospital Dublin. }\end{array}$ & $\begin{array}{l}\text { All women who delivered a baby weighing } \\
\geq 500 \mathrm{~g} \text { at Coombe Women and Infants } \\
\text { University Hospital Dublin. } \\
\mathrm{n}=38528 \text {. }\end{array}$ & $\begin{array}{l}\text { Occupation/ } \\
\text { social class. }\end{array}$ & $\begin{array}{l}\text { Preterm birth. } \\
\text { Spontaneous preterm } \\
\text { birth. } \\
\text { Elective preterm } \\
\text { birth. }\end{array}$ & $\begin{array}{l}6 \\
\text { High }\end{array}$ \\
\hline
\end{tabular}

\begin{tabular}{|c|c|c|c|c|c|c|}
\hline $\begin{array}{l}\text { Waterstone et al } \\
2001, \text { England }^{102}\end{array}$ & $\begin{array}{l}\text { Case-control study } \\
1997-1998\end{array}$ & $\begin{array}{l}\text { All } 19 \text { maternity units } \\
\text { within the South East } \\
\text { Thames region and } \\
\text { six neighbouring } \\
\text { hospitals. }\end{array}$ & $\begin{array}{l}\text { Cases were women from the South East } \\
\text { Thames region (and six neighbouring } \\
\text { hospitals) who delivered after } 24 \text { weeks' } \\
\text { gestation and who met the definition of } \\
\text { severe obstetric morbidity (severe pre- } \\
\text { eclampsia, severe haemorrhage, severe } \\
\text { sepsis and uterine rupture). Four controls } \\
\text { per case were selected randomly. } \\
\text { Cases: } n=588 \text {. } \\
\text { Controls: } n=48272 \text {. }\end{array}$ & $\begin{array}{l}\text { Occupation/ } \\
\text { social class. }\end{array}$ & $\begin{array}{l}\text { Severe maternal } \\
\text { morbidity. }\end{array}$ & $\begin{array}{l}5 \\
\text { Moderate }\end{array}$ \\
\hline $\begin{array}{l}\text { Wilding et al 2019, } \\
\text { England }^{82}\end{array}$ & $\begin{array}{l}\text { Cohort study 2004- } \\
2016\end{array}$ & $\begin{array}{l}\text { University Hospital } \\
\text { Southampton. }\end{array}$ & $\begin{array}{l}\text { Women aged } 18 \text { years or older who had } \\
\text { a live singleton birth at the University } \\
\text { Hospital Southampton National Health } \\
\text { Service Trust. } \\
n=43787 \text {. }\end{array}$ & $\begin{array}{l}\text { Education. } \\
\text { Employment. }\end{array}$ & $\begin{array}{l}\text { Small for gestational } \\
\text { age. }\end{array}$ & $\begin{array}{l}8 \\
\text { High }\end{array}$ \\
\hline $\begin{array}{l}\text { Wolke et al 2014, } \\
\text { England }^{87}\end{array}$ & $\begin{array}{l}\text { Case-control study } \\
1998-1999\end{array}$ & $\begin{array}{l}\text { Three maternity } \\
\text { units (and attached } \\
\text { neonatal intensive } \\
\text { care units) in } \\
\text { Southeast England. }\end{array}$ & $\begin{array}{l}\text { Infants who were very preterm (VP)/VLBW } \\
(<1500 \mathrm{~g} \text { or }<32 \text { weeks of gestation) and } \\
\text { admitted to one of three neonatal intensive } \\
\text { care units in southeast England. Of the } 214 \\
\text { survivors, } 112 \text { were randomly approached } \\
\text { and parents of } 90 \text { infants consented. Full- } \\
\text { term infants ( } 37-42 \text { weeks' gestation) from } \\
\text { the same maternity units were approached } \\
\text { in order to match to the VPNLBW sample } \\
\text { characteristics. } \\
\text { Cases: } n=90 \text {. } \\
\text { Controls: } n=115 \text {. }\end{array}$ & $\begin{array}{l}\text { Education. } \\
\text { Income. }\end{array}$ & $\begin{array}{l}\text { VLBW. } \\
\text { Very preterm birth. }\end{array}$ & $\begin{array}{l}4 \\
\text { Moderate }\end{array}$ \\
\hline
\end{tabular}

Dibben et al ${ }^{88}$ paper also reports VLBW and low birth weight by occupation/social status; however, as these data originate from the ONS (which is duplicated in the review), only income inequalities are reported here (see online supplemental appendix S8 for further details).

Data for ERHA reported annually between 1999 and 2001. However, data from 1999 only was used, as data for 2000 and 2001 (and subsequent years) was reported in Irish NPRS. ERHA is a former Health Board in the ROI, which encompasses people who live in Dublin, Wicklow and Kildare.

The data relating to the NPRS have been integrated to a single reference ${ }^{29}$; however, data originate from three annual reports produced over the period: 'Perinatal Statistics', ${ }^{34-37}$ the 'Report on Perinatal Statistics,38-42 and the 'Perinatal Statistics Report'. 43-52

Education and preterm birth for Niedhammer et $a l^{81}$ was not included as it is duplicated in Niedhammer et a $/^{91}$ (see online supplemental appendix S8 for further details). The data from the ONS have been integrated to a single reference; however, data originate from two annual reports: 'Mortality Statistics: Childhood, infant and perinatal, England and Wales'131-148 and 'Child mortality (death cohort) tables in England and Wales'. ${ }^{149-158}$

Education and preterm birth for Stacey et $a^{86}{ }^{6}$ was not included as it is duplicated in Poulsen et $a^{92}$ (see online supplemental appendix S8 for further details).

$\mathrm{ROI}$, Republic of Ireland.

supplemental appendix S12Aiii, Biii, Ciii). Sensitivity analysis demonstrated that the meta-analysis results were robust to the effect of any individual studies for all stillbirth analyses (online supplemental appendix S12Aiv) and for perinatal and neonatal mortality overall pooled meta-analyses and lowest versus highest occupation subgroups (online supplemental appendix S12Biv, Civ); however, the significance changed with the removal of the Office for National Statistics (ONS) 2002-2017 dataset in the intermediate subgroup for both outcomes. There was evidence of publication bias for stillbirth (online supplemental appendix S12Aii) but not for perinatal or neonatal mortality (online supplemental appendix S12Bii, Cii). One study ${ }^{28}$ not able to be pooled in the stillbirth meta-analysis reported increased odds of stillbirth among unemployed mothers (OR $1.24,95 \%$ CI 1.00 to 1.54 ) and fathers (OR 2.26, 95\% CI 1.69 to 3.02 ) compared with those who were employed (online supplemental appendix S11, table a). Maternal mortality was reported by one study $^{33}$ that showed a 


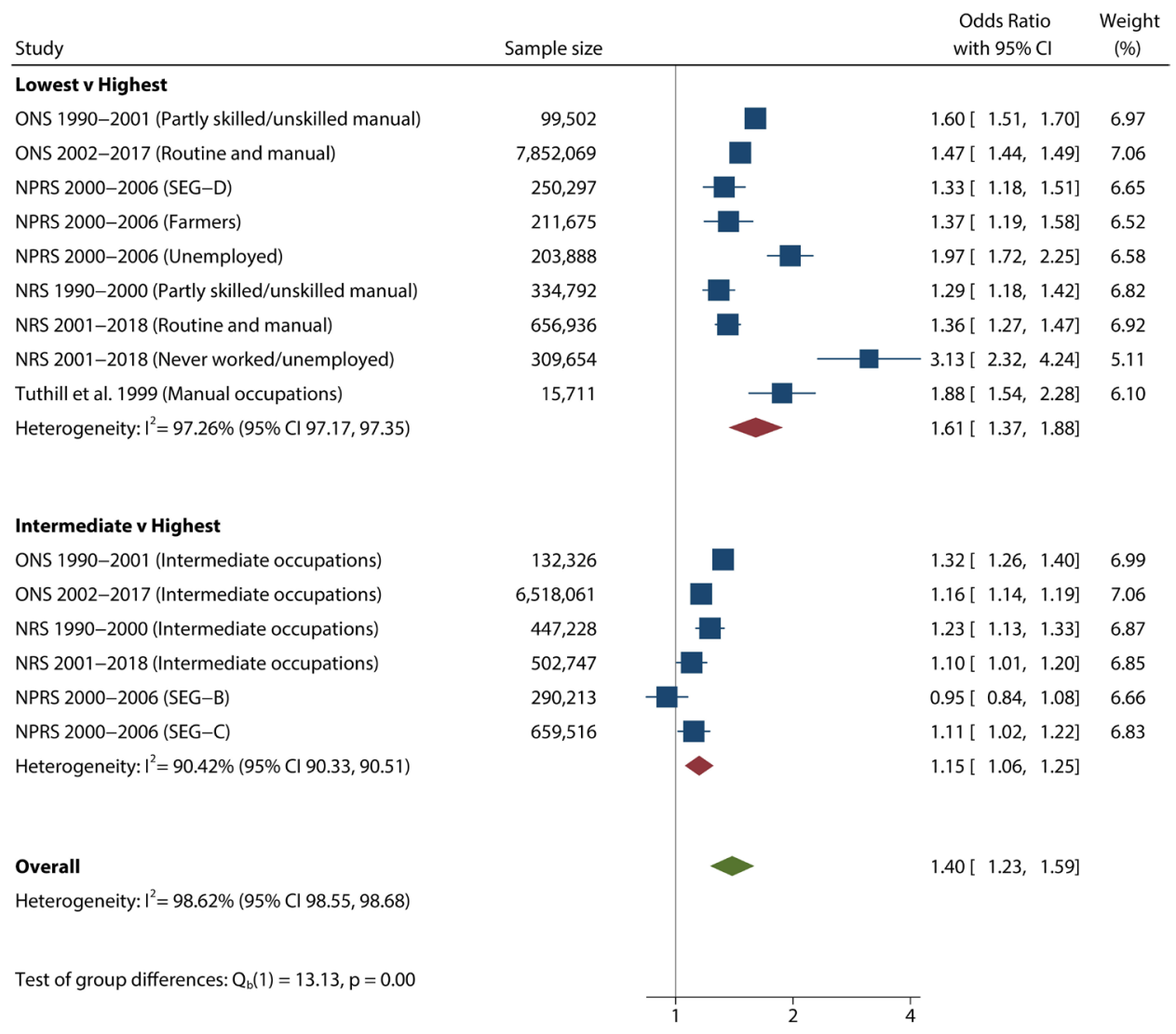

Figure 2 Forest plot of stillbirth. REML, Restricted Maximum Likelihood

significantly increased odds among unemployed women compared with those in employment following adjustment for confounding factors (AOR 1.81, 95\% CI 1.08 to 3.04, online supplemental appendix S11 table d). GRADE scores showed very low-quality evidence for stillbirth and low-quality of evidence for neonatal, perinatal

\begin{tabular}{|c|c|c|c|}
\hline Study & Sample size & $\begin{array}{l}\text { Odds Ratio } \\
\text { with } 95 \% \mathrm{Cl}\end{array}$ & $\begin{array}{l}\text { Weight } \\
(\%)\end{array}$ \\
\hline \multicolumn{4}{|l|}{ Intermediate v Highest } \\
\hline ONS 1990-2001 (Intermediate occupations) & 132,326 & $1.32[1.26,1.40]$ & 10.36 \\
\hline ONS 2002-2017 (Intermediate occupations) & $6,518,061$ & $1.18[1.16,1.21]$ & 10.54 \\
\hline NPRS 2000-2006 (SEG-B) & 290,213 & $1.00[0.91,1.11]$ & 9.87 \\
\hline NPRS 2000-2006 (SEG-C) & 659,516 & $1.15[1.06,1.24]$ & 10.14 \\
\hline Heterogeneity: $I^{2}=93.72 \%(95 \% \mathrm{Cl} 93.62,93.81)$ & & $1.17[1.05,1.30]$ & \\
\hline \multicolumn{4}{|l|}{ Lowest v Highest } \\
\hline ONS 1990-2001 (Partly skilled/unskilled manual) & 99,502 & $1.65[1.58,1.74]$ & 10.39 \\
\hline ONS 2002-2017 (Routine and manual) & $7,852,069$ & $1.49[1.46,1.51]$ & 10.55 \\
\hline NPRS 2000-2006 (SEG-D) & 250,297 & $1.35[1.22,1.50]$ & 9.85 \\
\hline NPRS 2000-2006 (Farmers) & 211,675 & $1.38[1.22,1.55]$ & 9.61 \\
\hline NPRS 2000-2006 (Unemployed) & 203,888 & $1.94[1.73,2.16]$ & 9.71 \\
\hline Tuthill et al. 1999 (Manual occupations) & 15,976 & $1.70[1.45,1.99]$ & 8.98 \\
\hline Heterogeneity: $\mathrm{I}^{2}=93.75 \%(95 \% \mathrm{Cl} 93.66,93.84)$ & & $1.57[1.41,1.74]$ & \\
\hline Overall & & $1.39[1.23,1.57]$ & \\
\hline \multicolumn{4}{|l|}{ Heterogeneity: $I^{2}=98.69 \%(95 \%$ Cl 98.62, 98.76) } \\
\hline \multicolumn{4}{|l|}{ Test of group differences: $Q_{b}(1)=14.62, p=0.00$} \\
\hline & & & \\
\hline
\end{tabular}

Figure 3 Forest plot of perinatal mortality. 


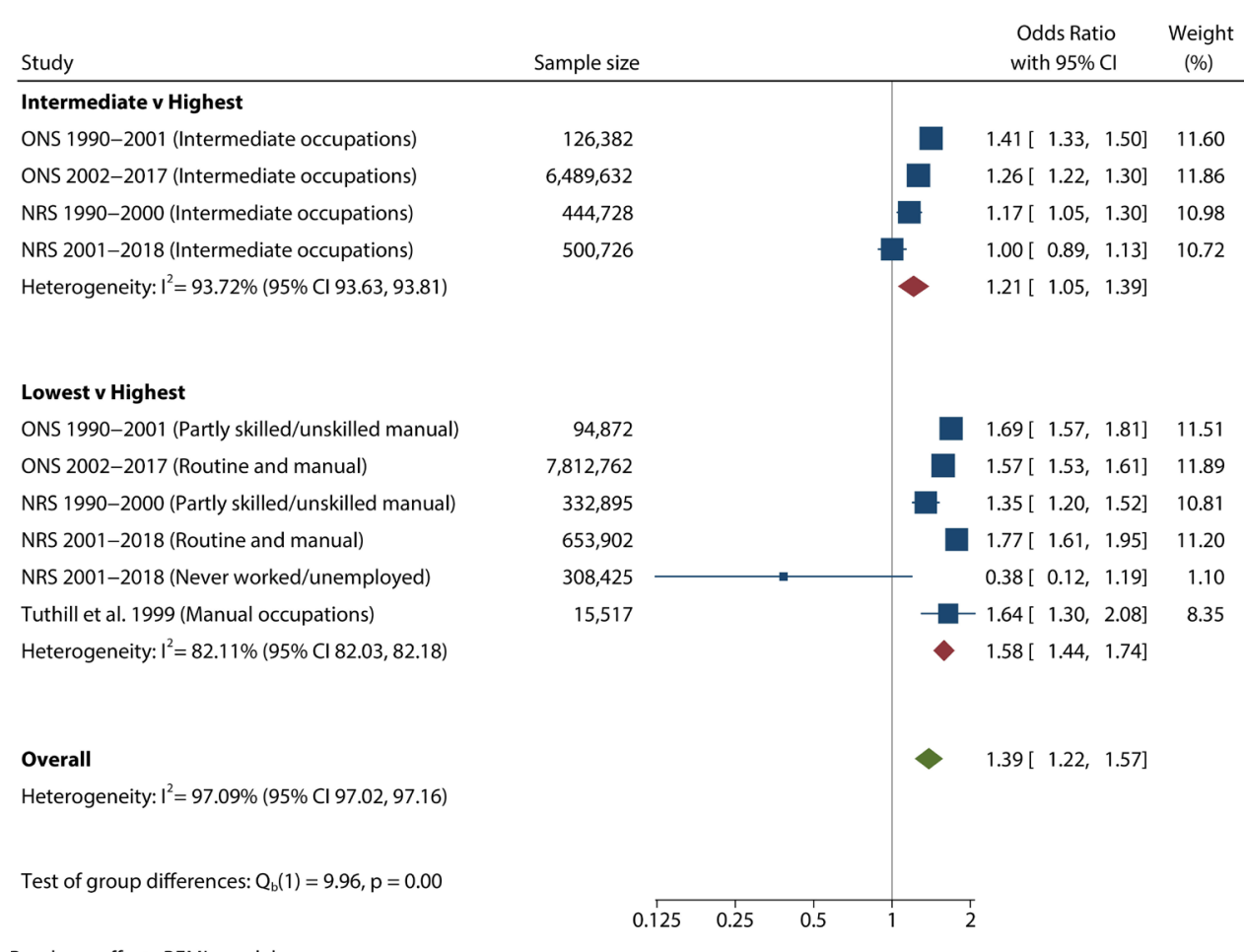

Figure 4 Forest plot of neonatal mortality.

and maternal mortality (online supplemental appendix S13).

\section{Birth weight}

Thirteen studies ${ }^{29} 31$ 34-78 reported associations between low and high birth weight outcomes and paternal and maternal occupation/social class, education, housing, income/household finances and car ownership. A metaanalysis was possible for occupation and very low birth weight (VLBW), LBW and macrosomia.

Two studies ${ }^{29} 31$ reported associations between occupation/social class and VLBW ( $<1500 \mathrm{~g}, \mathrm{n}=10284045$ births $)$ and macrosomia (>4000g, n=3430632 births) (online supplemental appendix S11, tables e and g), and both were included in the meta-analysis. Six studies reported LBW (<2500 g, n=3430632 births) with four ${ }^{29} 317980$ that could be included in the meta-analysis (online supplemental appendix S11, table f). The meta-analysis for the pooled lowest/intermediate occupation compared with highest identified no significant association with VLBW (OR $1.1895 \%$ CI 0.89 to 1.57), a significantly increased odds of LBW (OR $1.4095 \%$ CI 1.19 to 1.61 ) and decreased odds of macrosomia (OR $0.8995 \%$ CI 0.79 to 0.99 ) (figures 5-7). The subgroup meta-analysis identified that the highest odds for LBW were in the lowest level occupation category (OR $1.5595 \%$ CI 1.24 to 1.86 , figure 6 ), whereas for macrosomia, only the intermediatelevel versus highest level occupation remained significant (figure 7). There was significant heterogeneity in all meta-analyses for birthweight outcomes that were not explained by any of the factors included in metaregression (online supplemental appendix S12Diii, Eiii,
Fiii). There was no evidence of publication bias (online supplemental appendix S12Dii, Eii, Fii). Sensitivity analysis demonstrated that the results for birth weight metaanalysis were not robust. For LBW, results were robust for the overall pooled analysis and for the lowest level subgroup. However, the exclusion of datasets in the LBW intermediate-level subgroup and all macrosomia groupings resulted in the odds no longer being significant, whereas the exclusion of one dataset in the VLBW analysis resulted in the odds becoming significant for the pooled meta-analysis and both subgroups (online supplemental appendix S12Div, Eiv, Fiv). The GRADE score for LBW showed low-quality evidence (online supplemental appendix S13).

Additional data were reported for occupation and midlow birth weight (MLBW) $<3000 \mathrm{~g}^{29} 31$ and SGA $<5$ th or $<10$ th percentile ${ }^{79} 8182$ (online supplemental appendix S11, tables h and i). Predominantly, the data showed a significantly increased odds of SGA (following adjustment for confounding factors) and MLBW when mothers had lowest level occupations compared with highest, ${ }^{29} 317982$ whereas the evidence was conflicting for intermediate/ non-manual occupations. ${ }^{29} 317981$ No association was found between SGA and whether the mother had a contract post, worked shifts or long hours or had an active or stressful job. ${ }^{81}$

Further SES exposures reported for birthweight outcomes were education, ${ }^{81-87}$ income/finance ${ }^{83} 84$ 86-88 and overcrowding, housing tenure and car ownership, ${ }^{89}$ the majority of which adjusted for confounding factors. There was significantly increased odds of $\mathrm{LBW}^{83} \mathrm{SGA}^{82}$ 


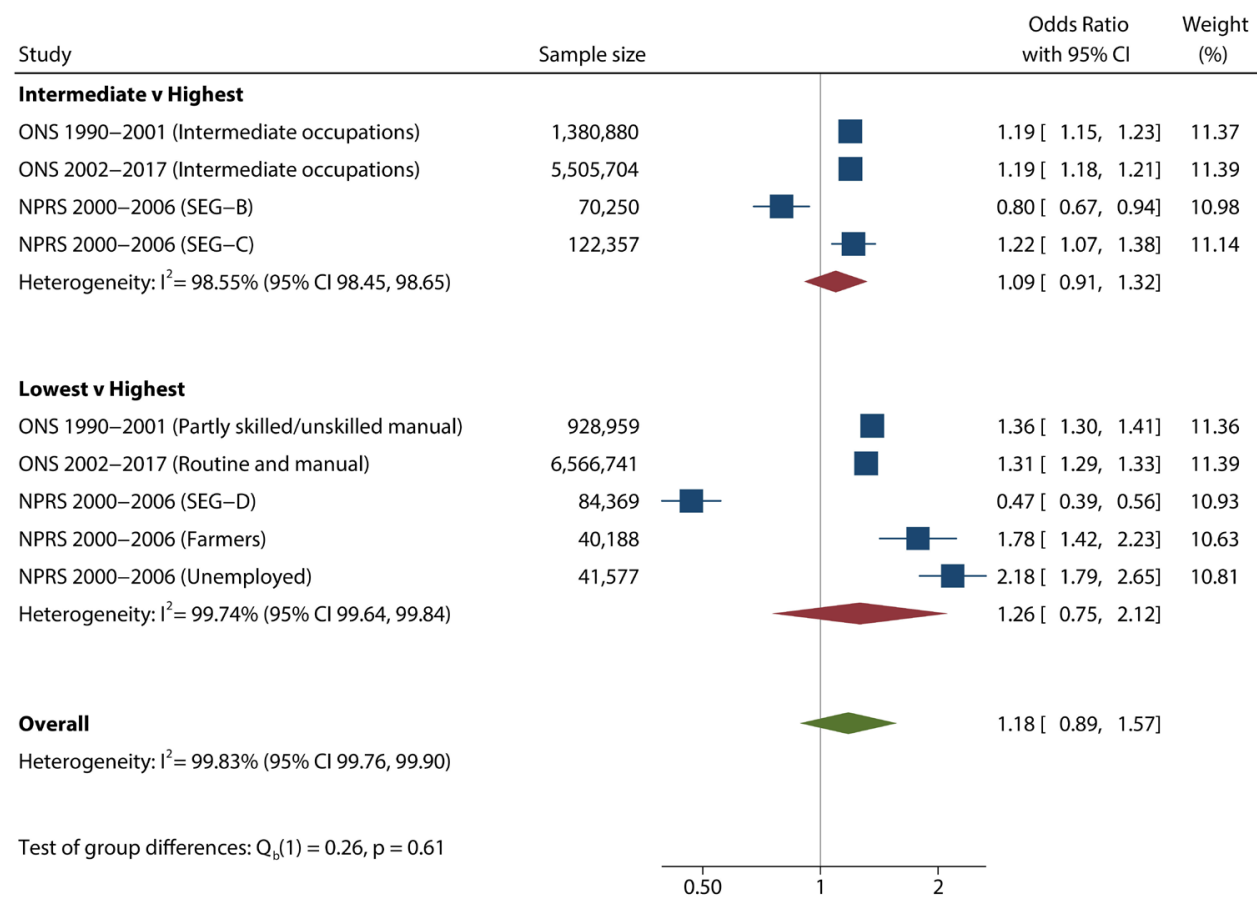

Figure 5 Forest plot of VLBW.

and $\mathrm{IUGR}^{84}$ for lower levels of education when studies used degree-level education as the comparison group (online supplemental appendix S11-table j); however, studies using other comparison groups reported no significant associations with high or LBWs. ${ }^{81}{ }^{85-87}$ There was significantly increased odds of LBW in lower income

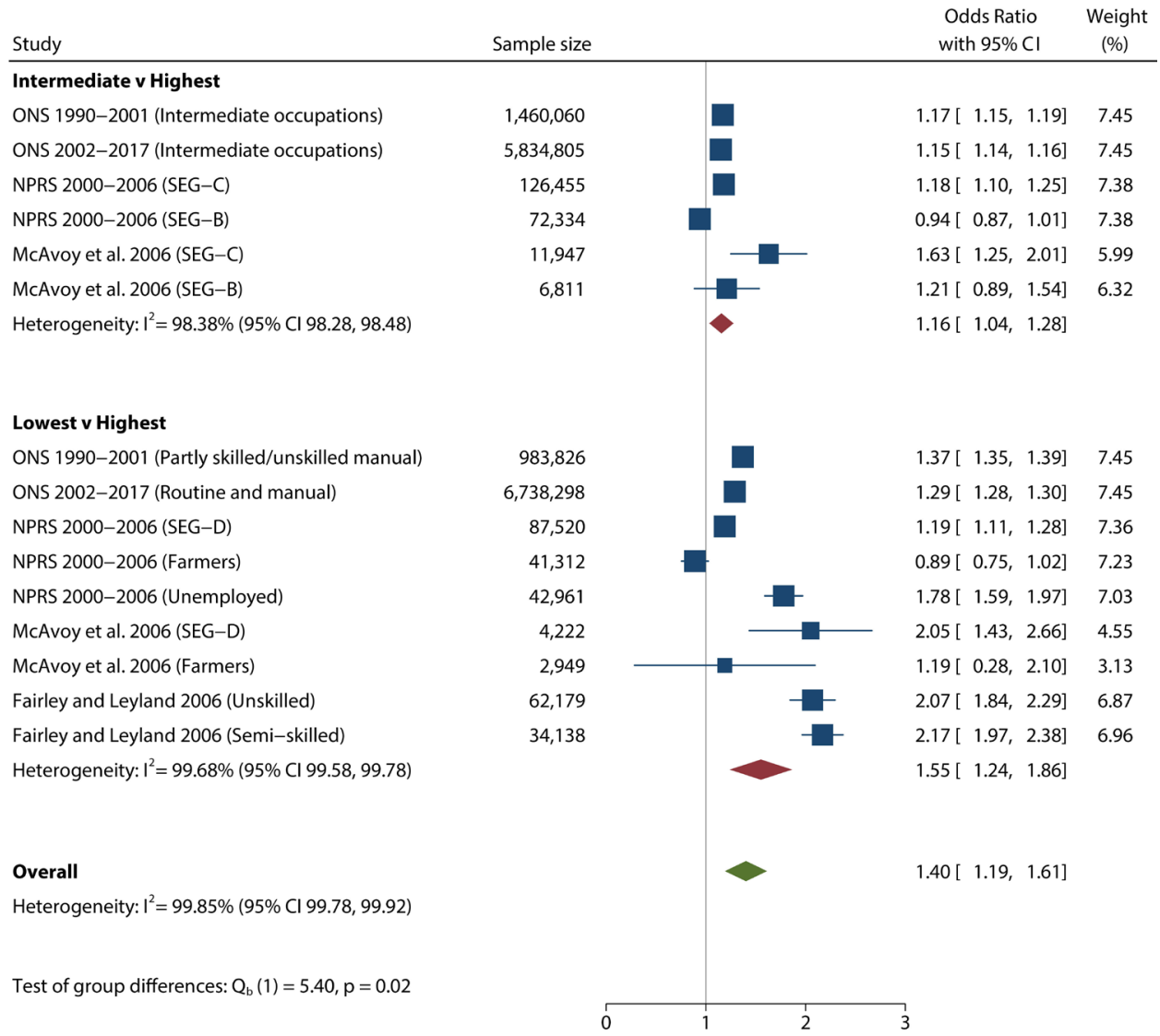

Figure 6 Forest plot of LBW. 


\begin{tabular}{|c|c|c|c|}
\hline Study & Sample size & $\begin{array}{l}\text { Odds ratio } \\
\text { with } 95 \% \mathrm{Cl}\end{array}$ & $\begin{array}{l}\text { Weight } \\
(\%)\end{array}$ \\
\hline \multicolumn{4}{|l|}{ Intermediate v Highest } \\
\hline ONS 1990-2001 (Intermediate occupations) & 365,386 & $0.87[0.85,0.88]$ & 11.20 \\
\hline ONS 2002-2017 (Intermediate occupations) & $1,641,611$ & $0.91[0.90,0.92]$ & 11.22 \\
\hline NPRS 2000-2006 (SEG-C) & 150,574 & $0.93[0.90,0.95]$ & 11.16 \\
\hline NPRS 2000-2006 (SEG-B) & 87,811 & $1.02[0.99,1.05]$ & 11.15 \\
\hline Heterogeneity: $I^{2}=97.75 \%(95 \% \mathrm{Cl} 97.57,97.93)$ & & $0.93[0.87,0.99]$ & \\
\hline \multicolumn{4}{|l|}{ Lowest v Highest } \\
\hline ONS 1990-2001 (Partly skilled/unskilled manual) & 264,801 & $0.77[0.75,0.79]$ & 11.17 \\
\hline ONS 2002-2017 (Routine and manual) & $2,143,915$ & $0.80[0.79,0.81]$ & 11.21 \\
\hline NPRS 2000-2006 (SEG-D) & 104,696 & $0.94[0.91,0.97]$ & 11.13 \\
\hline NPRS 2000-2006 (Farmers) & 50,374 & $1.22[1.16,1.29]$ & 10.97 \\
\hline NPRS 2000-2006 (Unemployed) & 51,035 & $0.65[0.61,0.70]$ & 10.79 \\
\hline Heterogeneity: $I^{2}=99.67 \%(95 \%$ Cl 99.50, 99.84) & & $0.86[0.70,1.05]$ & \\
\hline $\begin{array}{l}\text { Overall } \\
\text { Heterogeneity: } I^{2}=99.71 \%(95 \% \mathrm{Cl} 99.58,99.83)\end{array}$ & & $0.89[0.79,0.99]$ & \\
\hline \multicolumn{4}{|l|}{ Test of group differences: $Q_{b}(1)=0.56, p=0.45$} \\
\hline & $0.50 \quad 0.60$ & & \\
\hline
\end{tabular}

Random-effects REML model

Figure 7 Forest plot of macrosomia.

groups ${ }^{83} 88$ but not for $\mathrm{VLBW}^{87}$ or IUGR, ${ }^{84}$ or between other finance measures and $\mathrm{SGA}^{86}$ (online supplemental appendix S11, table k). One study ${ }^{89}$ reported a significantly increased odds of LBW when babies were born to mothers living in overcrowded households and in council/housing association properties but no significant associations with the number of rooms or renting (online supplemental appendix S11, table 1). One study ${ }^{89}$ reported a significantly increased association between LBW and households with no cars and a significantly decreased association for households with $>1$ car (online supplemental appendix S11, table m).

\section{Preterm birth}

Eight studies ${ }^{79} 8486$ 90-94 reported a combination of adjusted and unadjusted associations between PTB and occupation/social class, employment status, education, housing and income (online supplemental appendix S11, tables n-q). Meta-analysis was possible for the association between occupation/social class and preterm birth, which was reported by two studies ${ }^{79}$ for 515752 births (online supplemental appendix S11, table $\mathrm{n}$ ). The pooled metaanalysis for lowest/intermediate occupation compared with highest identified a significantly increased odds (OR $1.41,95 \%$ CI 1.33 to 1.50 ; figure 8 ). The subgroup metaanalysis identified that the highest odds for PTB were in the lowest level occupation category (OR 1.51, 95\% CI 1.42 to 1.60$)$. Heterogeneity was moderate for the lowest level occupation subgroup analysis but substantial for the overall meta-analysis and the intermediate occupation subgroup (figure 8), and these were not explained by the factors included in meta-regression (online supplemental appendix S12Giii). Sensitivity analysis demonstrated that the meta-analysis results were robust to the effect of any individual studies (online supplemental appendix S12Giv), and there was no evidence of publication bias (online supplemental appendix S12Gii). The GRADE score for preterm birth showed very low-quality evidence (online supplemental appendix S13).

Additional data reported for PTB included employment, education, housing and income or other finance measures. Two studies ${ }^{9394}$ reported significantly increased odds of PTB (overall, spontaneous and elective) among unemployed women compared with employed (online supplemental appendix S11, table n). Five studies ${ }^{840-93}$ reported an overall lack of association between education and PTB (online supplemental appendix S11, table o). Two studies ${ }^{8493}$ reported the association between income and PTB, and another ${ }^{86}$ reported the association between financial difficulties and PTB (online supplemental appendix S11, table p). For income, there was a significantly increased odds among women in the lowest income group ${ }^{84}$ in one study but not in another. ${ }^{93}$ There were also significantly increased odds among women 'not managing financially' but not for 'being behind with bills' ${ }^{86}$ A significantly increased risk of PTB was reported for babies born to women living in crowded homes compared with non-crowded homes, ${ }^{91}$ and there was conflicting evidence ${ }^{91} 93$ for rented housing (online supplemental appendix S11, table q).

\section{Mode of delivery}

Five studies reported the associations between occupation/social class and caesarean delivery (emergency, elective or any, a combination of unadjusted and adjusted data), ${ }^{95-98}$ instrumental delivery (all adjusted for 


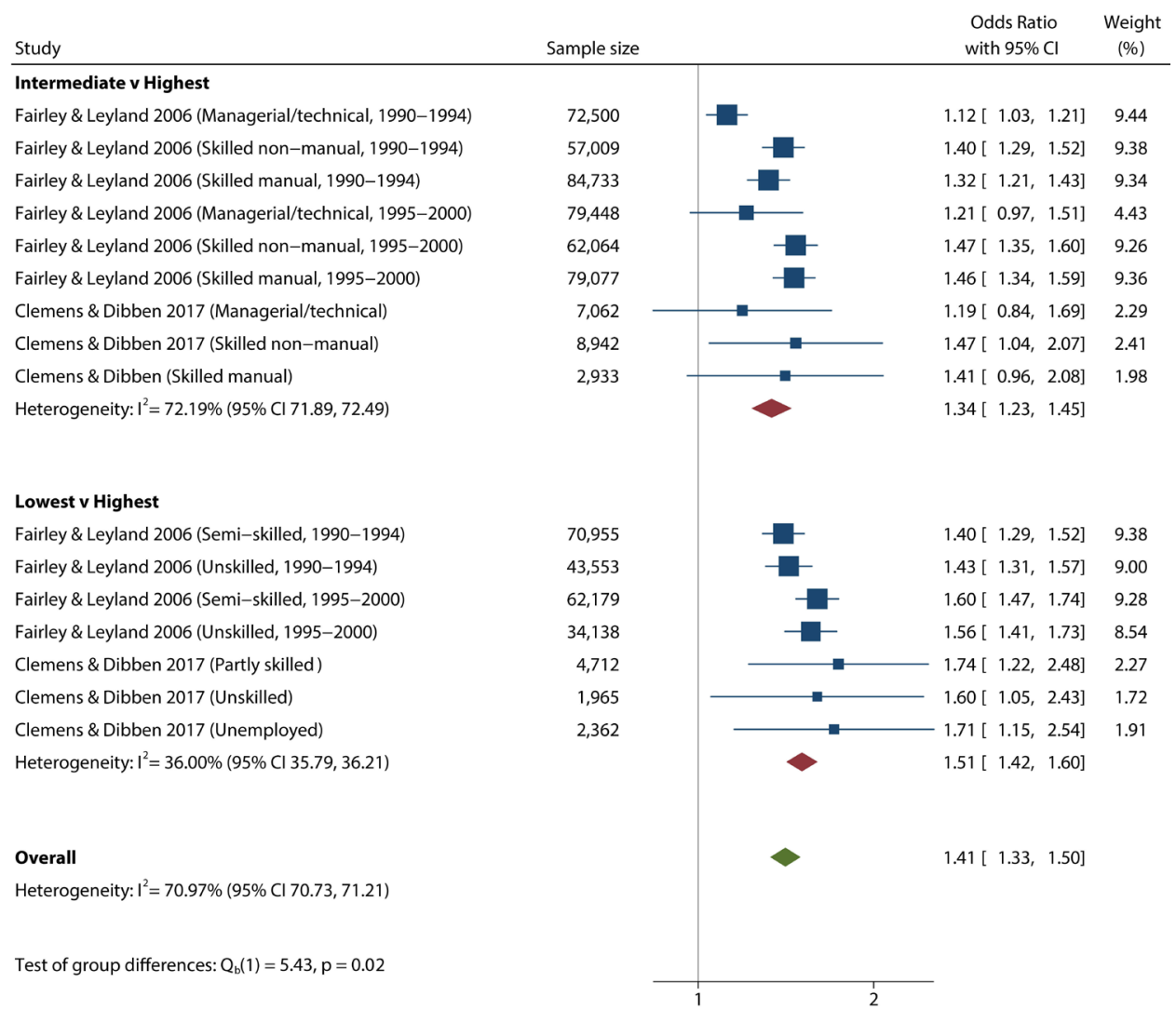

Figure 8 Forest plot for preterm birth ( $<37$ weeks gestation).

confounders) ${ }^{96}$ and induction (all unadjusted). ${ }^{99}$ Metaanalysis was possible for caesarean delivery. Any caesarean section (elective or emergency combined) was reported by three studies ${ }^{959698}$ for 50345 births, whereas emergency and elective caesarean were reported separately by four studies ${ }^{95-98}$ for $>47496$ births (sample size not reported for one study, ${ }^{97}$ online supplemental appendix S11, table $\mathrm{r}$ ), and all were included in the meta-analysis. The meta-analysis identified a significantly reduced odds of emergency caesarean (OR $0.85,95 \%$ CI 0.77 to 0.94 ; figure 9), elective caesarean (OR $0.83,95 \%$ CI 0.76 to 0.91 ; figure 10) and any caesarean (OR $0.80,95 \% \mathrm{CI}$ 0.74 to 0.86 ; figure 11) for the pooled lowest/intermediate occupation/social class compared with the highest level. The subgroup meta-analysis identified that the lowest occupation/social class had the lowest odds of all caesarean categories (figures 9-11). There was moderate heterogeneity for any caesarean delivery, and this was significantly reduced in the subgroup analysis (figure 11). Heterogeneity was moderate for elective caesarean, which was reduced by subgrouping (figure 10) and further partly explained by the date of data collection (online supplemental appendix S12Iiii). There was high heterogeneity for emergency caesarean pooled data, which was partly explained by subgrouping (figure 9) and further explained to a very small degree by sample size but not by the other factors included in meta-regression (online supplemental appendix 12Hii). Sensitivity analysis demonstrated that the meta-analysis results were robust to the effect of individual studies (online supplemental appendix S12Hiv, Iiv, Jiv), and there was no evidence of publication bias for any category of caesarean section (online supplemental appendix S12Hii, Iii, Jii). The GRADE score for caesarean section showed low-quality evidence (online supplemental appendix S13).

Additional data for caesarean delivery identified a significantly decreased odds among women in the lowest income quintile ${ }^{84}$ (online supplemental appendix S11, table s), living in council housing, housing association or privately rented accommodation (online supplemental appendix S11, table t) and among women who bent or stood a lot and physically exerted themselves at work ${ }^{98}$ but an increased odds among women who mostly sat, undertook demanding tasks, worked during pregnancy and worked more than 50 hours (online supplemental appendix S11, table v) ${ }^{98}$ There was an overall lack of association with maternal education ${ }^{8496}$ (online supplemental appendix S11, table $u$ ). There was limited data for instrumental delivery ${ }^{96}$ and induction ${ }^{99}$ (online supplemental appendix S11, tables $\mathrm{w}-\mathrm{x}$ ) with some conflicting results depending on parity and previous mode of delivery.

\section{Other outcomes reported}

Six studies reported no significant associations between occupation and placental problems, ${ }^{100}$ maternal morbidity, ${ }^{101} 102$ myocardial infarction, ${ }^{103}$ antenatal stroke $^{104}$ or amniotic fluid embolism ${ }^{105}$ (online supplemental appendix S11, table y). One study reported 


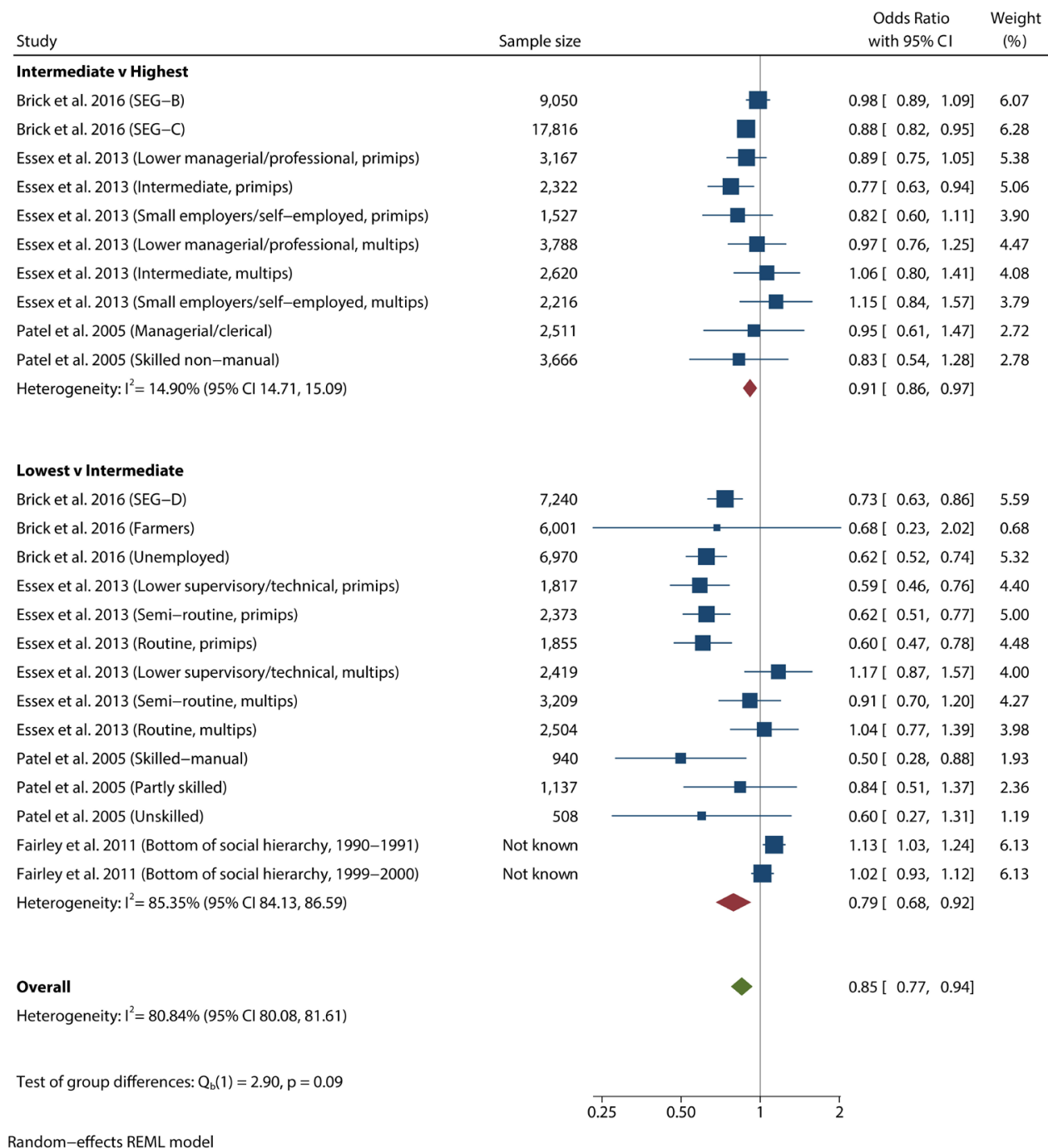

Figure 9 Forest plot of emergency caesarean section.

associations between education level and congenital anomalies ${ }^{106}$ with significantly increased odds of congenital anomalies for educational attainment levels below a diploma, degree or higher degree qualification (online supplemental appendix S11, table z). All data were unadjusted for confounding factors.

\section{DISCUSSION}

Meta-analysis from this systematic review of 17130631 births in the UK and ROI identified that lower level occupation/social class is associated with a significantly increased risk of stillbirth, neonatal mortality, perinatal mortality, preterm birth and LBW by approximately $40 \%$. Additional data narratively summarised suggest there is also an increased risk of stillbirth, maternal mortality and preterm birth among unemployed parents. Further individual level SES factors with some, although limited, evidence of increased risk of adverse pregnancy outcomes include lowest level of education (compared with women who have a degree), housing and income.
Overall, the data on individual and household measures of inequality suggest women (and families) experiencing lower SES face generally worse pregnancy outcomes compared with women who have a higher social status. Education, housing, income, taxation and social protection policies have undermined social mobility, leading to widening and persistent inequalities. ${ }^{1}$ Austerity has been associated with widening inequalities in a variety of health outcomes, including infant mortality rate. ${ }^{2} 107 \mathrm{In}$ response to economic hardship, women are less likely to adopt positive health-related behaviours due to the need to focus on coping in the short term rather than planning for the future. ${ }^{108}$ As such, poverty experienced by those in lower SES might also be a pathway leading to worse pregnancy outcomes alongside poorer nutrition, housing insecurity, financial stress ${ }^{2}$ and the wider social determinants of health. These associations may be mediated by health behaviours and other risk factors, whereby mothers facing economic hardship are, for example, more likely to smoke during pregnancy. Our analysis has demonstrated a relationship between low SES and PTB (which increases 


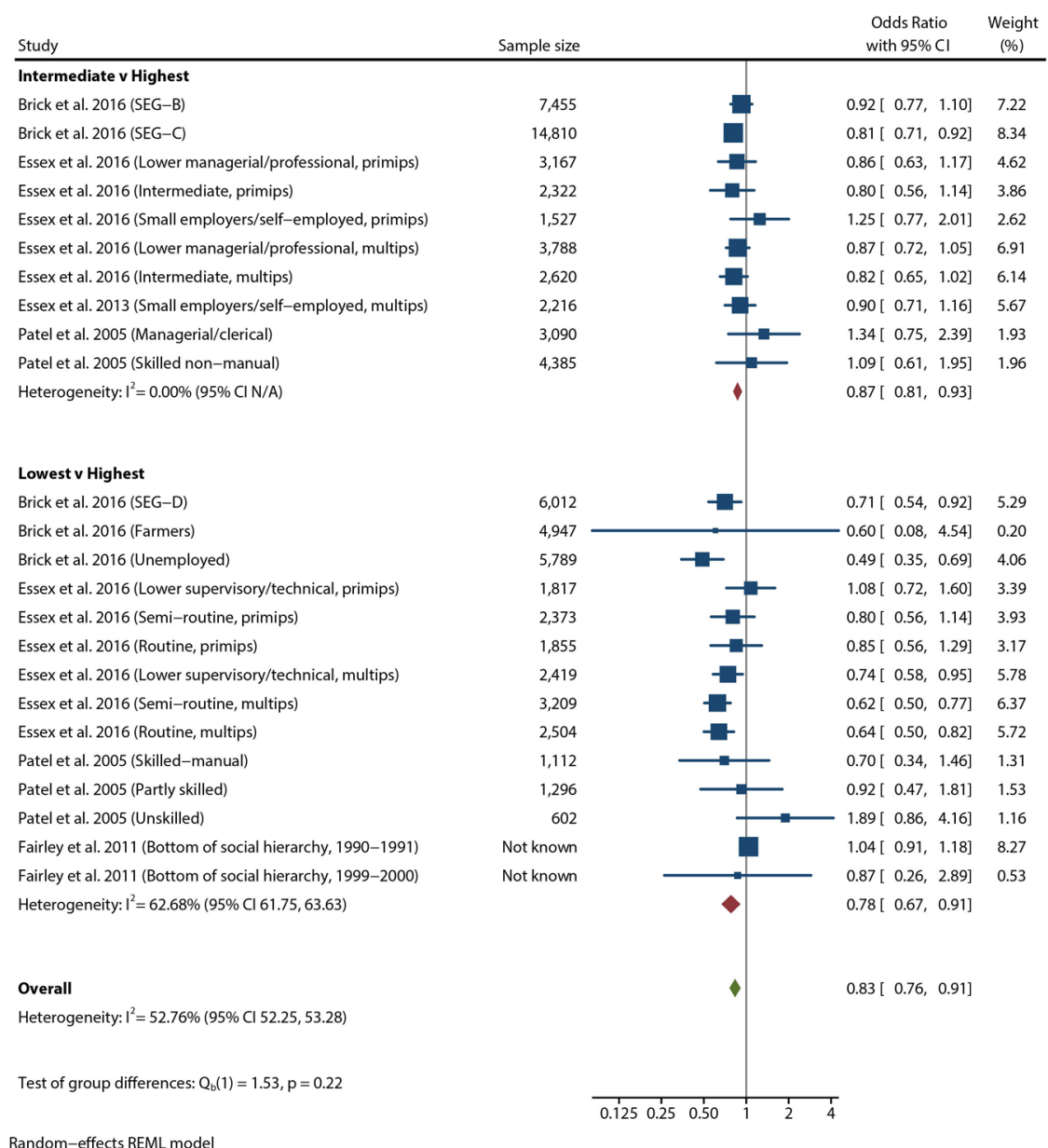

Figure 10 Forest plot of elective caesarean section.

the risk of subsequent infant mortality), and this is also related to low maternal weight and smoking. ${ }^{82}$ Furthermore, deprivation is related to poor diet, ${ }^{109}$ low levels of physical activity, ${ }^{110}$ increased obesity ${ }^{111}$ late access to (and low engagement with) antenatal care, ${ }^{112}$ increased drug ${ }^{113}$ and alcohol use, ${ }^{114}$ and exposure to domestic violence. ${ }^{115}$ Tackling the fundamental drivers of poor pregnancy outcomes is imperative as they are associated with lower educational attainment and disadvantage throughout the lifecourse, ${ }^{116} 117$ therefore, contributing to the intergenerational cycle of inequality. This has implications considering the post-COVID-19 economic recovery, and the need for governments to not follow austerity again and instead focus on policies that will not further exacerbate inequalities, particularly for women and children.

This review has many strengths as well as limitations. Our search strategy was broad and wide ranging, which included multiple databases supplemented by searching citations and reference lists and contacting experts in each of the four UK regions and the ROI. The grey literature sources resulted in several large datasets being included from national records in all nations of the UK and the ROI. Using national datasets, supplemented by additional academic publications, has resulted in large sample sizes for some outcome/SES combinations, particularly for occupation and for stillbirths, neonatal mortality, PTB and birth weight. We did not restrict by language, and all screening, data extraction and quality assessments were carried out in duplicate to minimise human error. We also did not restrict by pregnancy outcome; consequently, we have wide ranging outcomes highlighting the effects of SES on the health of the mother and baby during pregnancy and up to 28 days postnatal. However, we did have to exclude studies that reported perinatal mental health or breastfeeding outcomes due to the lack of clarity in reporting the time periods of measurement for these outcomes and therefore these require further exploration capturing a longer postnatal period than 28 days. Using a validated quality appraisal tool, all included studies were rated moderate to high quality. However, our findings refer to UK and ROI only, and therefore, further research should be undertaken to enable an understanding of how pregnancy outcomes relate to socioeconomic determinants across other HICs. Furthermore, rich data sources from the ONS and National Records of Scotland were used extensively in this review to document the association between occupation-derived social class and birth weight, stillbirth, neonatal and perinatal mortality. Over the 28 years for which we had data, the occupational classifications evolved in line with changing labour market conditions as did the reporting of birth registrations; therefore, the categories with which we analysed data 


\begin{tabular}{|c|c|c|c|c|}
\hline Study & Sample size & & $\begin{array}{r}\text { Odds Ratio } \\
\text { with } 95 \% \mathrm{Cl}\end{array}$ & $\begin{array}{c}\text { Weight } \\
(\%)\end{array}$ \\
\hline \multicolumn{5}{|l|}{ Intermediate v Highest } \\
\hline Brick et al. 2016 (SEG-B) & 9,663 & & $0.97[0.88,1.06]$ & 7.07 \\
\hline Brick et al. 2016 (SEG-C) & 18,898 & & $0.87[0.81,0.93]$ & 7.47 \\
\hline Essex et al. 2013 (Lower managerial/professional, primips) & 3,167 & & $0.87[0.74,1.02]$ & 5.85 \\
\hline Essex et al. 2013 (Intermediate, primips) & 2,322 & & $0.76[0.63,0.91]$ & 5.36 \\
\hline Essex et al. 2013 (Small employer/self-employed, primips) & 1,527 & & $0.91[0.69,1.20]$ & 3.84 \\
\hline Essex et al. 2013 (Lower managerial/professional, multips) & 3,788 & & $0.90[0.76,1.05]$ & 5.81 \\
\hline Essex et al. 2013 (Intermediate, multips) & 2,620 & & $0.89[0.74,1.07]$ & 5.30 \\
\hline Essex et al. 2013 (Small employer/self-employed, multips) & 2,216 & & $0.99[0.80,1.22]$ & 4.94 \\
\hline Patel et al. 2005 (Managerial/clerical) & 3,090 & & $1.08[0.75,1.54]$ & 2.84 \\
\hline Patel et al. 2005 (Skilled non-manual) & 4,385 & & $0.93[0.65,1.32]$ & 2.89 \\
\hline Heterogeneity: $I^{2}=16.52 \%(95 \% \mathrm{Cl} 16.32,16.72)$ & & $\diamond$ & $0.90[0.85,0.95]$ & \\
\hline \multicolumn{5}{|l|}{ Lowest v Highest } \\
\hline Brick et al. 2016 (SEG-D) & 7,721 & & $0.73[0.63,0.84]$ & 6.25 \\
\hline Brick et al. 2016 (Farmers) & 6,417 & & $0.67[0.25,1.80]$ & 0.53 \\
\hline Brick et al. 2016 (Unemployed) & 7,422 & - & $0.59[0.50,0.70]$ & 5.78 \\
\hline Essex et al. 2013 (Lower supervisory/technical, primips) & 1,817 & $\rightarrow-$ & $0.67[0.53,0.84]$ & 4.57 \\
\hline Essex et al. 2013 (Semi-routine, primips) & 2,373 & - & $0.63[0.53,0.77]$ & 5.31 \\
\hline Essex et al. 2013 (Routine, primips) & 1,855 & $\neg$ & $0.63[0.50,0.79]$ & 4.61 \\
\hline Essex et al. 2013 (Lower supervisory/technical, multips) & 2,419 & - & $0.88[0.72,1.07]$ & 5.11 \\
\hline Essex et al. 2013 (Semi-routine, multips) & 3,209 & - & $0.70[0.59,0.83]$ & 5.51 \\
\hline Essex et al. 2013 (Routine, multips) & 2,504 & 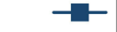 & $0.75[0.62,0.92]$ & 5.09 \\
\hline Patel et al. 2005 (Skilled-manual) & 1,112 & & $0.57[0.36,0.90]$ & 2.01 \\
\hline Patel et al. 2005 (Partly skilled) & 1,296 & & $0.92[0.62,1.38]$ & 2.40 \\
\hline Patel et al. 2005 (Unskilled) & 602 & & $1.07[0.62,1.87]$ & 1.49 \\
\hline Heterogeneity: $I^{2}=34.00 \%(95 \% \mathrm{Cl} 33.53,34.48)$ & & $\bullet$ & $0.70[0.65,0.76]$ & \\
\hline Overall & & $\diamond$ & $0.80[0.74,0.86]$ & \\
\hline \multicolumn{5}{|l|}{ Heterogeneity: $I^{2}=69.78 \%(95 \% \mathrm{Cl} 69.14,70.43)$} \\
\hline \multicolumn{5}{|l|}{ Test of group differences: $Q_{b}(1)=23.88, p=0.00$} \\
\hline & 0.25 & 0.50 & & \\
\hline
\end{tabular}

Figure 11 Forest plot of any caesarean section.

are not directly comparable. Broad groupings of lowest, intermediate and higher level occupations were required to be able to pool data, which may not reflect any differences within these groups (eg, any differences between unemployed vs manual employment that would have been grouped as lowest level). Additionally, the use of national datasets such as ONS required the calculation of effect sizes using aggregate frequency data provided in the reports; therefore, we were unable to account for potential confounding variables. There was inconsistency in the reporting of adjusted data throughout, where occupation-derived social class included mostly adjusted data for SGA and instrumental delivery, some adjusted data for LBW, preterm and caesarean delivery, and a lack of adjusted data for other outcomes such as offspring mortality, VLBW and macrosomia. However, most education, income/finances and housing data were adjusted, although reported to a lesser extent overall. We had intended to explore adjustment for confounding variables in the meta-regression as an a priori factor; however, this was not possible due to the limited availability of adjusted data for occupation-derived social class used in the metaanalyses. There were some outcomes where both unadjusted and adjusted data were available (eg, LBW online supplemental appendix S11f). Comparing these, the effect sizes and significance were similar in the univariable and multivariable models that included multiple sociodemographic and behavioural factors. There are some additional limitations with the use of routine data sources such as issues of accuracy of data recorded in routine health records and the comparability and granularity of variables between datasets. However, routine datasets tend to be nationally representative and adequately powered to explore patterns in population health, in a timely manner. These strengths are especially important for research where outcomes are relatively rare, such as stillbirth and congenital anomalies, which would require extensive funding and time to collect prospectively. In addition, for research into health inequalities, the use of routine data are a timely method of identifying potentially vulnerable groups who require additional support. Alternative methods that incorporate lengthy data collection periods could be considered unethical when alternative existing data sources are available.

This review is subject to the usual limitations of observational research whereby we cannot claim that there is a causal relationship between individual and household measures of socioeconomic disadvantage and pregnancy outcomes. As such, the quality of evidence highlighted by GRADE was typically low or very low. Although outcomes were downgraded (eg, for inconsistency as heterogeneity was high for all meta-analyses), due to the use of national datasets showing large effects, upgrading also occurred. We have also only found a limited number of studies for some outcome/ 
SES combinations, and therefore, it is possible that there is publication bias (that negative results are less likely to be published) and selective reporting within studies that may impact the assessment of risk of bias. To negate this, where possible, we calculated associations between inequality exposures and pregnancy outcomes when these had not been published (eg, if frequency data were reported in a paper but no association or by using frequency data published in nationally representative datasets such as ONS). In the metaanalysis, only stillbirth had significant evidence of publication bias. The positive associations with stillbirth may therefore be an artefact of significant associations being more likely to be published as only one reported association included in the meta-analysis was not significant. However, we should also consider the sample sizes that contributed to these significant associations, the largest being provided by the ONS (2002-17) and including $>7.8$ million women in the comparison of lowest versus highest occupation-derived social class and $>6.5$ million women in the intermediate versus highest comparison. There are some methodological deviations from the published protocol. First, we limited studies to the UKand ROI only, due to the volume of studies from European HICs that we retrieved in the search. Second, we anticipated only including a meta-analysis if there were three of more studies reporting associations between the same exposure and outcome. However, we made the decision to include metaanalysis of two studies in the final paper due to the limited amount of data available for some combinations of exposure and outcome. Most of these studies reported multiple effect sizes for subgroups of participants, which could contribute to the overall pooled estimate and be valuable to explore underreported exposure and outcome combinations.

While there was substantial evidence for some SES measures, there were very limited data available for housing, income and car ownership, and a lack of data for other measures of individual or household inequality that may be important including other measures of poverty and social isolation. Furthermore, there was a lack of consistency in definitions used between studies, particularly for education, which made direct comparisons difficult, and it was not possible to pool these data in a meta-analysis. Future studies should explore these other axes of inequalities in addition to gain a broader insight into the relative importance and interplay of socioeconomic variables and pregnancy outcomes. Similarly, although we did identify a range of pregnancy outcomes, there was an absence of evidence available for other outcomes that require further exploration, such as miscarriage, postpartum haemorrhage, gestational diabetes, hypertensive disorders of pregnancy, measures of newborn health (eg, Apgar score), attachment and the need for high-risk healthcare services (eg, neonatal intensive care). This systematic review did not include pregnancy outcomes beyond 28 days postnatal, such as breast feeding, postnatal depression and infant mortality. These additional inequality measures and health outcomes warrant further research to supplement the existing evidence base and inform public health and maternity policy and practice, particularly in light of rising inequalities in infant mortality in the UK. ${ }^{2}$

The early-life health outcomes and exposure to SES inequalities identified in this review have life-long consequences; for example, the prevalence of severe mental health problems is around three times higher among children in the bottom quintile of family income compared with those in the top quintile. ${ }^{118}$ Over the last 10years, austerity measures have impacted those poorest in our society the most. As a result of tax and welfare policies, between 2010/2011 and $2015 / 2016$, the number of people in work and living in poverty has increased to 3.7 million. ${ }^{119}$ Furthermore, funding for supporting community services such as Sure Start Children's Centres has been widely cut, despite having been shown to reduce hospitalisations and improve inequalities, including reducing SES inequalities in infant mortality. ${ }^{120}$ Indeed, research found that SES inequalities in infant mortality decreased during a period of social policy investment in England (the English ealth inequalities strategy 2000-2010). ${ }^{107}$ Consequently, more children are born into poverty with associated inequalities in health outcomes such as those identified in this review, as well as infant mortality that disproportionally impacts deprived communities. ${ }^{2}$

This study looked specifically for socioeconomic inequalities in pregnancy outcomes in the UK and Ireland alone. These countries have broadly comparable inequalities, which are typically higher compared with other parts of Europe owing to a minimal welfare state. ${ }^{121}$ Cross-country analysis does, however, suggest there may be important differences in pregnancy outcomes ${ }^{21011}$ and health more generally. ${ }^{122}$ Although evidence for occupation/social class differences was greatest in this review, there has in recent times been a move to reframe health inequalities away from individual 'labels' to instead focus on power relations and to interrogate the processes that produce them. ${ }^{123}$ As such, identifying inequalities during pregnancy is a step towards informing where services could adapt provision to effectively support populations that are socioeconomically disadvantaged. However, targeting interventions solely at the most disadvantaged groups will not reduce the steepness of the social gradient; for example, we identified that women in the intermediate-level occupations were also at increased risk of some adverse pregnancy outcomes. Proportionate universalism instead advocates a universal approach with a scale and intensity that is proportionate to the level of disadvantage. ${ }^{124}$ This requires engagement between health professionals and people from lower socioeconomic groups in shared decision making, which is too often characterised by less information giving, less directions and less socioemotional and partnership building. ${ }^{125}$ Our review demonstrated that lower SES women are less likely to have a caesarean section, which could be protective against the increased harm associated with an operative birth; however, this could highlight that women from deprived communities are less likely to request a caesarean, or more worryingly, be involved in discussions around birthing options. A greater consciousness of structural constraints, and a willingness by health professionals to systematically break them down, may empower marginalised, 
lower socioeconomic patients to be more involved in decisions around their care and help reduce inequalities in the long term.

At a policy level, this review highlights that employment and workplace policies need to consider their role in supporting better pregnancy outcomes. The period of employment covers the years when people are starting and raising families and as such is instrumental in reducing the transmission of inequities to the next generation. ${ }^{1}$ Yet austerity has driven fundamental shifts in the labour market and a push towards employment, whatever its quality or stability, leading to more precarious and part-time opportunities. Since the financial crisis, these changes have given rise to a reduction in real earnings, which for families is exacerbated further by a large reduction in benefits available for working age people and children. ${ }^{126}$ Indeed, USA research suggests that pushing low-income mothers into work has little health benefit. ${ }^{127}$ Through shaping policies related to healthcare, public health and social policy, governments can influence the social determinants of health. ${ }^{128}$ Social protection policies as well as active labour market policies and family policies may help reduce inequalities, although the evidence currently available is inconclusive. ${ }^{126} 129$

This review has highlighted that systematic collection of routine data in pregnancy allows for associations between upstream socioeconomic factors and pregnancy outcomes to be established as evidenced in the included large national datasets for occupation-derived social class and various pregnancy outcomes. Thus, improvements to the quality and completeness of routinely collected national datasets in the antenatal and perinatal period could have a powerful impact on policy development.

Overall, the findings from this review highlight that pregnancy outcomes are also associated with factors that lie upstream of the health behaviours of women and the care they receive in pregnancy and labour. These findings support the need for 'whole system' approaches to explore and address socioeconomic inequalities and pregnancy outcomes. Key areas for action may include: improved surveillance; analysis of the policy landscape impacting pregnant women and their families, with consideration of the role of different sectors and settings (including the National Health Service); and timely and rigorous research and evaluation efforts to further develop understanding of population needs for pregnant women and their families and the ways to address these needs.

\section{CONCLUSION}

The findings from this review provide evidence that there is a worsening of health for both babies and pregnant women who experience higher levels of individual and household socioeconomic deprivation. The strongest current evidence base is for increased risk of mortality, LBW and PTB among lower level occupation-derived social classes. Strategies to improve pregnancy outcomes should consider this evidence base and incorporate approaches that address the wider determinants of health to provide women and their families with the best chance of having a healthy pregnancy and baby and to decrease pregnancy-related health inequalities in the general population.

Acknowledgements The project is a collaboration between Public Health Wales and Fuse (UKCRC Centre for Translational Research Public Health). NH, HB, CB and $J R$ are members of Fuse, the Centre for Translational Research in Public Health ( www.fuse.ac.uk). Fuse is a Public Health Research Centre of Excellence funded by the five North East Universities of Durham, Newcastle, Northumbria, Sunderland and Teesside. We would like to thank our colleagues at Public Health Wales, Frank Dixon (National Records of Scotland) and Leslie Marr (The Scottish Health Council) for their help with regards sourcing grey literature. We would also like to thank authors of the studies who provided additional data including Aoife Brick (Economic and Social Research Institute) and Chris Dibben (University of Edinburgh).

Contributors AM, AJ, JB, SW, CB, HB, NH and JR conceived the study idea and developed the proposal. $C R$ developed the search strategy with contributions from $\mathrm{KT}, \mathrm{OA}, \mathrm{NH}, \mathrm{JR}, \mathrm{CB}, \mathrm{AJ}$ and $\mathrm{SW}$. CR, KT, OA and $\mathrm{AO}$ carried out the database searches and supplementary searches. $\mathrm{KT}, \mathrm{OA}, \mathrm{MM}, \mathrm{AO}$ and $\mathrm{NH}$ completed the screening, data extraction and quality appraisals. MM ran the meta-analysis with support from NH and HB. KT and NH wrote the first draft of the manuscript. All authors edited drafts of this article and approved the final version. The corresponding author attests that all listed authors meet authorship criteria and that no others meeting the criteria have been omitted. $\mathrm{NH}$ is the guarantor.

Funding The review was commissioned by Public Health Wales. Three public health consultants from Public Health Wales are authors on the paper.

Competing interests All authors have completed the ICMJE uniform disclosure form at www.icmje.org/coi_disclosure.pdf and declare: support from Public Health Wales for the submitted work; AJ, SW, JB and AM are all employees of Public Health Wales.

Patient consent for publication Not required.

Provenance and peer review Not commissioned; externally peer reviewed.

Data availability statement All data relevant to the study are included in the article or uploaded as supplementary information.

Supplemental material This content has been supplied by the author(s). It has not been vetted by BMJ Publishing Group Limited (BMJ) and may not have been peer-reviewed. Any opinions or recommendations discussed are solely those of the author(s) and are not endorsed by BMJ. BMJ disclaims all liability and responsibility arising from any reliance placed on the content. Where the content includes any translated material, BMJ does not warrant the accuracy and reliability of the translations (including but not limited to local regulations, clinical guidelines, terminology, drug names and drug dosages), and is not responsible for any error and/or omissions arising from translation and adaptation or otherwise.

Open access This is an open access article distributed in accordance with the Creative Commons Attribution Non Commercial (CC BY-NC 4.0) license, which permits others to distribute, remix, adapt, build upon this work non-commercially, and license their derivative works on different terms, provided the original work is properly cited, appropriate credit is given, any changes made indicated, and the use is non-commercial. See: http://creativecommons.org/licenses/by-nc/4.0/.

ORCID iD

Katie Thomson http://orcid.org/0000-0002-9614-728X

\section{REFERENCES}

1 Marmot M. Health equity in England: the Marmot review 10 years on. BMJ 2020;368:m693.

2 Taylor-Robinson D, Lai ETC, Wickham S, et al. Assessing the impact of rising child poverty on the unprecedented rise in infant mortality in England, 2000-2017: time trend analysis. BMJ Open 2019:9:e029424.

3 Ludvigsson JF, Lu D, Hammarström L, et al. Small for gestational age and risk of childhood mortality: a Swedish population study. PLoS Med 2018;15:e1002717.

4 Boghossian NS, Geraci M, Edwards EM, et al. Morbidity and mortality in small for gestational age infants at 22 to 29 weeks gestation. Pediatrics 2018;141:e20172533.

5 Callaghan WM, MacDorman MF, Rasmussen SA, et al. The contribution of preterm birth to infant mortality rates in the United States. Pediatrics 2006;118:1566-73. 
6 Vos AA, Posthumus AG, Bonsel GJ, et al. Deprived neighborhoods and adverse perinatal outcome: a systematic review and metaanalysis. Acta Obstet Gynecol Scand 2014;93:727-40.

7 Blumenshine P, Egerter S, Barclay CJ, et al. Socioeconomic disparities in adverse birth outcomes: a systematic review. Am J Prev Med 2010;39:263-72.

8 O'Connell R, Owen C, Padley M, et al. Which types of family are at risk of food poverty in the UK? A relative deprivation approach. Soc Policy Soc 2019;18:1-18.

9 Bambra C, Riordan R, Ford J. The COVID-19 pandemic and health inequalities. J Epidemiol Community Health 2020;121:jech-2020214401.

10 Global Health Observatory. Child mortality levels: number of deaths (by country), 2019. Available: http://apps.who.int/gho/data/node. main.ChildMort-1 ?lang=en [Accessed 14 Oct 2019].

11 World Health Organization U, UNFPA, World Bank Group and UNPD (MMEIG). Maternal mortality, 2019. Available: https://data.unicef. org/topic/maternal-health/maternal-mortality/

$12 \mathrm{Kim} \mathrm{D,} \mathrm{Saada} \mathrm{A.} \mathrm{The} \mathrm{social} \mathrm{determinants} \mathrm{of} \mathrm{infant} \mathrm{mortality}$ and birth outcomes in Western developed nations: a crosscountry systematic review. Int J Environ Res Public Health 2013;10:2296-335.

13 Amjad S, MacDonald I, Chambers T, et al. Social determinants of health and adverse maternal and birth outcomes in adolescent pregnancies: a systematic review and meta-analysis. Paediatr Perinat Epidemiol 2019;33:88-99.

14 Weightman AL, Morgan HE, Shepherd MA, et al. Social inequality and infant health in the UK: systematic review and meta-analyses. BMJ Open 2012;2:e000964.

15 Moher D, Liberati A, Tetzlaff J, et al. Preferred reporting items for systematic reviews and meta-analyses: the PRISMA statement. PLoS Med 2009;6:e1000097.

16 Mackenbach JP. The English strategy to reduce health inequalities. Lancet 2011;377:1986-8.

17 Stroup DF, Berlin JA, Morton SC. Meta-analysis of observational studies in epidemiology a proposal for reporting. JAMA 2000;283:2008-12.

18 McKenzie JE, Brennan SE, Ryan RE. Defining the criteria for including studies and how they will be grouped for the synthesis. In: Higgins J, Thomas J, Chandler J, eds. Cochrane Handbook for systematic reviews of interventions version 60, 2019.

19 Clarivate Analytics. Endnote X8.2 [program]. Philadelphia, USA: Clarivate Analytics, 2019

20 Veritas Health Innovation. Covidence systematic review software, 2019. Available: www.covidence.org

21 Wells GA, Shea B, O'Connell D. The Newcastle-Ottawa scale (NOS) for assessing the quality of nonrandomised studies in metaanalyses, 2019. Available: http://www.ohri.ca/programs/clinical_ epidemiology/oxford.asp

22 Guyatt GH, Oxman AD, Vist GE, et al. Grade: an emerging consensus on rating quality of evidence and strength of recommendations. BMJ 2008;336:924-6.

23 Balshem H, Helfand M, Schünemann $\mathrm{HJ}$, et al. Grade guidelines: 3 . rating the quality of evidence. J Clin Epidemiol 2011;64:401-6.

24 Higgins JPT, Thompson SG, Deeks JJ, et al. Measuring inconsistency in meta-analyses. BMJ 2003;327:557-60.

25 StataCorp. Stata statistical software: release 16. College Station, TX: StataCorp LLC, 2019.

26 Popay J, Roberts H, Sowden A. Guidance on the conduct of narrative synthesis in systematic reviews: a product from the ESRC methods programme. 92. Lancaster: Lancaster University, 2006.

27 Office for National Statistics. Standard occupational classification 2010: volume 3 the National statistics socio-economic classification: (Rebased on the SOC2010) user manual. Palgrave Macmillan, 2010.

28 Gardosi J, Madurasinghe V, Williams M, et al. Maternal and fetal risk factors for stillbirth: population based study. BMJ 2013;346:f108.

29 National Perinatal Reporting System. Perinatal statistics. Dublin: Republic of Ireland, 1993: 1999-2016.

30 National Records of Scotland. Numbers of births, stillbirths and neonatal deaths, registered in Scotland in each year from 1990 to 2018 [bespoke request]. Edinburgh: National Records of Scotland, 2019.

31 Office for National Statistics. Mortality statistics: childhood, infant and perinatal, England and Wales (1990 - 2007) and Child mortality (death cohort) tables in England and Wales (2008 - 2017). Newport, Wales, 2017

32 Tuthill DP, Stewart JH, Coles EC, et al. Maternal cigarette smoking and pregnancy outcome. Paediatr Perinat Epidemiol 1999;13:245-53.

33 Nair M, Knight M, Kurinczuk JJ. Risk factors and newborn outcomes associated with maternal deaths in the UK from 2009 to 2013: a national case-control study. BJOG: Int J Obstet Gy 2016;123:1654-62.

34 Department of Health. Perinatal statistics 1990. Dublin: Department of Health, 1990.

35 Do H. Perinatal statistics. Dublin: Department of Health, 1991.

36 Department of Health Management Information Unit. Perinatal statistics 1992. Dublin: Department of Health, 1992.

37 Department of Health and Children. Perinatal statistics 1993. Dublin: Department of Health and Children, 1993.

38 Cullen G. Report on perinatal statistics for 1999. Dublin: The Economic and Social Research Institute, 2002.

39 Bonham S. Report on perinatal statistics for 2000. Dublin: Economic and Social Research Institute, 2004.

40 Bonham S. Report on perinatal statistics for 2001. Dublin: Economic and Social Research Institute, 2005

41 Bonham S. Report on perinatal statistics for 2003. Dublin: Economic and Social Research Institute, 2006.

42 Bonham S. Report on perinatal statistics for 2004. Dublin: Economic and Social Research Institute, 2007.

43 Health Research and Information Division. Perinatal statistics report 2005. Dublin: Economic and Social Research Institute, 2008.

44 Health Research and Information Division. Perinatal statistics report 2007. Dublin: Economic and Social Research Institute, 2009.

45 Health Research and Information Division. Perinatal statistics report 2008. Dublin: Economic and Social Research Institiute, 2010.

46 Health Research and Information Division. Perinatal statistics report 2009. Dublin: Economic and Social Research Institute, 2011.

47 Health Research and Information Division. Perinatal statistics report 2010. Dublin: Economic and Social Research Institute, 2012.

48 Health Research and Information Division. Perinatal statistics report 2012. Dublin: Economic and Social Research Institute, 2013.

49 Healthcare Pricing Office (HPO). Perinatal statistics report, 2013. Dublin, 2014.

50 Healthcare Pricing Office (HPO). Perinatal statistics report, 2014. Dublin, 2016.

51 Healthcare Pricing Office (HPO). Perinatal statistics report, 2015. Dublin, 2017.

52 Healthcare Pricing Office (HPO). Perinatal statistics report, 2016. Dublin, 2018.

53 Office of National Statistics. Mortality statistics: childhood, infant and perinatal, England and Wales (Series DH3) - No. 39, 2006 London: Office of National Statistics, 2008.

54 Office of National Statistics. Mortality statistics: childhood, infant and perinatal, England and Wales (Series DH3) - No. 40, 2007. London: Office of National Statistics, 2009.

55 Office of National Statistics. Child mortality (death cohort) tables in England and Wales. London: Office of National Statistics, 2010.

56 Office of National Statistics. Child mortality (death cohort) tables in England and Wales. London: Office of National Statistics, 2011.

57 Office of National Statistics. Child mortality (death cohort) tables in England and Wales. London: Office of National Statistics, 2012.

58 Office of National Statistics. Child mortality (death cohort) tables in England and Wales. London: Office of National Statistics, 2013.

59 Office of National Statistics. Child mortality (death cohort) tables in England and Wales. London: Office of National Statistics, 2014.

60 Office of National Statistics. Child mortality (death cohort) tables in England and Wales. London: Office of National Statistics, 2015.

61 Office of National Statistics. Child mortality (death cohort) tables in England and Wales. London: Office of National Statistics, 2016.

62 Office of National Statistics. Child mortality (death cohort) tables in England and Wales. London: Office of National Statistics, 2017.

63 Office of National Statistics. Mortality statistics: childhood, infant and perinatal, England and Wales (Series DH3) - No. 32, 1999. London: Office of National Statistics, 2001.

64 Office of National Statistics. Mortality statistics: childhood, infant and perinatal, England and Wales (Series DH3) - No. 33, 2000. London: Office of National Statistics, 2002.

65 Office of National Statistics. Mortality statistics: childhood, infant and perinatal, England and Wales (Series DH3) - No. 34, 2001. London: Office of National Statistics,, 2003.

66 Office of National Statistics. Mortality statistics: childhood, infant and perinatal, England and Wales (Series DH3) - No. 35, 2002. London: Office of National Statistics, 2004.

67 Office of National Statistics. Mortality statistics: childhood, infant and perinatal, England and Wales (Series DH3) - No. 36, 2003. London: Office of National Statistics, 2005

68 Office of National Statistics. Mortality statistics: childhood, infant and perinatal, England and Wales (Series DH3) - No. 37, 2004. London: Office of National Statistics, 2006. 
69 Office of National Statistics. Mortality statistics: childhood, infant and perinatal, England and Wales (Series DH3) - No. 38, 2005. London: Office of National Statistics, 2007.

70 Office of National Statistics. Mortality statistics: childhood, infant and perinatal, England and Wales (series DH3), 1990. London: Office of National Statistics, 1992.

71 Office of National Statistics. Mortality statistics: childhood, infant and perinatal, England and Wales (series DH3), 1991. London: Office of National Statistics, 1993.

72 Office of National Statistics. Mortality statistics: childhood, infant and perinatal, England and Wales (series DH3), 1992. London: Office of National Statistics, 1994.

73 Office of National Statistics. Mortality statistics: childhood, infant and perinatal, England and Wales (series DH3), 1993. London: Office of National Statistics, 1995.

74 Office of National Statistics. Mortality statistics: childhood, infant and perinatal, England and Wales (series DH3), 1994. London: Office of National Statistics, 1996

75 Office of National Statistics. Mortality statistics: childhood, infant and perinatal, England and Wales (series DH3), 1995. London: Office of National Statistics, 1997.

76 Office of National Statistics. Mortality statistics: childhood, infant and perinatal, England and Wales (series DH3), 1996. London: Office of National Statistics, 1998.

77 Office of National Statistics. Mortality statistics: childhood, infant and perinatal, England and Wales (series DH3), 1997. London: Office of National Statistics, 1999.

78 Office of National Statistics. Mortality statistics: childhood, infant and perinatal, England and Wales (series DH3), 1998. London: Office of National Statistics, 2000.

79 Fairley L, Leyland $\mathrm{AH}$. Social class inequalities in perinatal outcomes: Scotland 1980-2000. J Epidemiol Community Health 2006;60:31-6.

80 McAvoy H, Sturley J, Burke S. Unequal at birth: inequalities in the occurrence of low birthweight babies in Ireland. 96. Dublin/Belfast: Institute of Public Health in Ireland, 2006.

81 Niedhammer I, O'Mahony D, Daly S, et al. Occupational predictors of pregnancy outcomes in Irish working women in the Lifeways cohort. BJOG 2009;116:943-52.

82 Wilding S, Ziauddeen N, Roderick P, et al. Are socioeconomic inequalities in the incidence of small-for-gestational-age birth narrowing? findings from a population-based cohort in the South of England. BMJ Open 2019;9:e026998.

83 Martinson ML, Reichman NE. Socioeconomic inequalities in low birth weight in the United States, the United Kingdom, Canada, and Australia. Am J Public Health 2016;106:748-54.

84 Matijasevich A, Victora CG, Lawlor DA, et al. Association of socioeconomic position with maternal pregnancy and infant health outcomes in birth cohort studies from Brazil and the UK. $J$ Epidemiol Community Health 2012;66:127-35.

85 Murrin C, Segonds-Pichon A, Fallon UB, et al. Self-reported prepregnancy maternal body mass index and infant birth-weight. Ir Med J 2007;100:20-3.

86 Stacey T, Prady S, Haith-Cooper M, et al. Ethno-specific risk factors for adverse pregnancy outcomes: findings from the born in Bradford cohort study. Matern Child Health J 2016;20:1394-404.

87 Wolke D, Eryigit-Madzwamuse S, Gutbrod T. Very preterm/very low birthweight infants' attachment: infant and maternal characteristics. Arch Dis Child Fetal Neonatal Ed 2014;99:F70-5

88 Dibben C, Sigala M, Macfarlane A. Area deprivation, individual factors and low birth weight in England: is there evidence of an "area effect"? J Epidemiol Community Health 2006;60:1053-9.

89 Collingwood Bakeo A, Clarke L. Risk factors for low birthweight based on birth registration and census information, England and Wales, 1981-2000. Health Stat Q 2006:15-21.

90 Clemens T, Dibben C. Living in stressful neighbourhoods during pregnancy: an observational study of crime rates and birth outcomes. Eur J Public Health 2017;27:197-202.

91 Niedhammer I, Murrin C, O'Mahony D, et al. Explanations for social inequalities in preterm delivery in the prospective Lifeways cohort in the Republic of Ireland. Eur J Public Health 2012;22:533-8.

92 Poulsen G, Strandberg-Larsen K, Mortensen L, et al. Exploring educational disparities in risk of preterm delivery: a comparative study of 12 European birth cohorts. Paediatr Perinat Epidemiol 2015;29:172-83.

93 Snelgrove JW, Murphy KE. Preterm birth and social inequality: assessing the effects of material and psychosocial disadvantage in a UK birth cohort. Acta Obstet Gynecol Scand 2015;94:766-75.

94 Vinturache A, McKeating A, Daly N, et al. Maternal body mass index and the prevalence of spontaneous and elective preterm deliveries in an Irish obstetric population: a retrospective cohort study. BMJ Open 2017;7:e015258.

95 Brick A, Layte R, Nolan A, et al. Differences in nulliparous caesarean section rates across models of care: a decomposition analysis. BMC Health Serv Res 2016;16:239-39.

96 Essex HN, Green J, Baston H, et al. Which women are at an increased risk of a caesarean section or an instrumental vaginal birth in the UK: an exploration within the millennium cohort study. BJOG 2013;120:732-43.

97 Fairley L, Dundas R, Leyland $\mathrm{AH}$. The influence of both individual and area based socioeconomic status on temporal trends in caesarean sections in Scotland 1980-2000. BMC Public Health 2011;11:330-30.

98 Patel RR, Peters TJ, Murphy DJ, et al. Prenatal risk factors for caesarean section. analyses of the ALSPAC cohort of 12,944 women in England. Int J Epidemiol 2005;34:353-67.

99 Sinnott S-J, Layte R, Brick A, et al. Variation in induction of labour rates across Irish hospitals; a cross-sectional study. Eur J Public Health 2016;26:753-60.

100 Fitzpatrick KE, Sellers S, Spark P, et al. Incidence and risk factors for placenta accreta/increta/percreta in the UK: a national casecontrol study. PLoS One 2012;7:e52893.

101 Nair M, Kurinczuk JJ, Knight M. Ethnic variations in severe maternal morbidity in the UK- a case control study. PLoS One 2014;9:e95086.

102 Waterstone M, Bewley S, Wolfe C. Incidence and predictors of severe obstetric morbidity: case-control study. BMJ 2001;322:1089-94.

103 Bush N, Nelson-Piercy C, Spark P, et al. Myocardial infarction in pregnancy and postpartum in the UK. Eur J Prev Cardiol 2013;20:12-20.

104 Scott CA, Bewley S, Rudd A, et al. Incidence, risk factors, management, and outcomes of stroke in pregnancy. Obstet Gynecol 2012;120:318-24.

105 Fitzpatrick KE, Tuffnell D, Kurinczuk JJ, et al. Incidence, risk factors, management and outcomes of amniotic-fluid embolism: a population-based cohort and nested case-control study. BJOG: Int J Obstet Gy 2016;123:100-9.

106 Sheridan E, Wright J, Small N, et al. Risk factors for congenital anomaly in a multiethnic birth cohort: an analysis of the born in Bradford study. Lancet 2013:382:1350-9.

107 Robinson T, Brown H, Norman PD, et al. The impact of new labour's English health inequalities strategy on geographical inequalities in infant mortality: a time-trend analysis. J Epidemiol Community Health 2019;73:564-8.

108 Bell R. Psychosocial pathways and health outcomes: informing action on health inequalities. London: The Institute of Health Equity, 2017.

109 Haggarty P, Campbell DM, Duthie S, et al. Diet and deprivation in pregnancy. Br J Nutr 2009;102:1487-97.

110 Muktabhant B, Lawrie TA, Lumbiganon P, et al. Diet or exercise, or both, for preventing excessive weight gain in pregnancy. Cochrane Database Syst Rev 2015;6:257.

111 Heslehurst N, Vieira R, Akhter Z, et al. The association between maternal body mass index and child obesity: a systematic review and meta-analysis. PLoS Med 2019;16:e1002817.

112 McDonagh M. Is antenatal care effective in reducing maternal morbidity and mortality? Health Policy Plan 1996;11:1-15.

113 Bauer CR, Shankaran S, Bada HS, et al. The maternal lifestyle study: drug exposure during pregnancy and short-term maternal outcomes. Am J Obstet Gynecol 2002;186:487-95.

114 Huizink AC, Mulder EJH, smoking M. Maternal smoking, drinking or cannabis use during pregnancy and neurobehavioral and cognitive functioning in human offspring. Neurosci Biobehav Rev 2006;30:24-41.

115 Janssen PA, Holt VL, Sugg NK, et al. Intimate partner violence and adverse pregnancy outcomes: a population-based study. Am J Obstet Gynecol 2003;188:1341-7.

116 Barker DJ, Forsén T, Uutela A, et al. Size at birth and resilience to effects of poor living conditions in adult life: longitudinal study. BM 2001;323:1273-6.

117 Johnson S, Wolke D, Hennessy E, et al. Educational outcomes in extremely preterm children: neuropsychological correlates and predictors of attainment. Dev Neuropsychol 2011;36:74-95.

118 Green H, McGinnity A, Ford T. Mental health of children and young people in Great Britain, 2004. London: Office for National Statistics 2005.

119 Joseph Rowntree Foundation. UK poverty. York: Joseph Rowntree Foundation, 2017.

120 Catten S, Conti G, Farquharson C. The health effects of sure start. London: Institute for Fiscal Studies, 2019. 
121 Eikemo TA, Bambra C, Joyce K, et al. Welfare state regimes and income-related health inequalities: a comparison of 23 European countries. Eur J Public Health 2008;18:593-9.

122 Balaj M, McNamara CL, Eikemo TA, et al. The social determinants of inequalities in self-reported health in Europe: findings from the European social survey (2014) special module on the social determinants of health. Eur J Public Health 2017;27:107-14

123 Gkiouleka A, Huijts T, Beckfield J, et al. Understanding the micro and macro politics of health: Inequalities, intersectionality \& institutions - A research agenda. Soc Sci Med 2018;200:92-8.

124 Marmot M, Allen J, Goldblatt P. Fair society healthy lives (the Marmot review). 242. London: Institute of Health Equity, University College London, 2010.

125 Willems S, De Maesschalck S, Deveugele M, et al. Socio-economic status of the patient and doctor-patient communication: does it make a difference? Patient Educ Couns 2005;56:139-46.

126 Clarke SCN. Setting the record straight: how record employment has changed the UK. London: Resolution Foundation, 2019.

127 Gibson M, Thomson H, Banas K, et al. Welfare-to-work interventions and their effects on the mental and physical health of lone parents and their children. Cochrane Database Syst Rev 2018;2:CD009820.

128 Bambra C, Gibson M, Sowden A, et al. Tackling the wider social determinants of health and health inequalities: evidence from systematic reviews. J Epidemiol Community Health 2010;64:284-91.

129 Hillier-Brown F, Thomson K, Mcgowan V, et al. The effects of social protection policies on health inequalities: evidence from systematic reviews. Scand J Public Health 2019;47:655-65.

130 Knight M, UKOSS. Antenatal pulmonary embolism: risk factors, management and outcomes. BJOG 2008;115:453-61.

131 Office for National Statistics. Mortality Statistics: Childhood, infant and perinatal, England and Wales (Series DH3) - No. 40, 2007. London: Office of National Statistics, 2009.

132 Office for National Statistics. Mortality Statistics: Childhood, infant and perinatal, England and Wales (Series DH3) - No. 39, 2006. London: Office of National Statistics, 2008.

133 Office for National Statistics. Mortality Statistics: Childhood, infant and perinatal, England and Wales (Series DH3) - No. 38, 2005. London: Office of National Statistics, 2007.

134 Office for National Statistics. Mortality Statistics: Childhood, infant and perinatal, England and Wales (Series DH3) - No. 37, 2004. London: Office of National Statistics, 2006.

135 Office for National Statistics. Mortality Statistics: Childhood, infant and perinatal, England and Wales (Series DH3) - No. 36, 2003. London: Office of National Statistics, 2005.

136 Office for National Statistics. Mortality Statistics: Childhood, infant and perinatal, England and Wales (Series DH3) - No. 35, 2002. London: Office of National Statistics, 2004.

137 Office for National Statistics. Mortality Statistics: Childhood, infant and perinatal, England and Wales (Series DH3) - No. 34, 2001. London: Office of National Statistics, 2003.
138 Office for National Statistics. Mortality Statistics: Childhood, infant and perinatal, England and Wales (Series DH3) - No. 33, 2000. London: Office of National Statistics, 2002.

139 Office for National Statistics. Mortality Statistics: Childhood, infant and perinatal, England and Wales (Series DH3) - No. 32, 1999. London: Office of National Statistics, 2001.

140 Office for National Statistics. Mortality statistics: childhood, infant and perinatal, England and Wales (series DH3), 1998. London: Office of National Statistics, 2000.

141 Office for National Statistics. Mortality statistics: childhood, infant and perinatal, England and Wales (series DH3), 1997. London: Office of National Statistics, 1999.

142 Office for National Statistics. Mortality statistics: childhood, infant and perinatal, England and Wales (series DH3), 1996. London: Office of National Statistics, 1998.

143 Office for National Statistics. Mortality statistics: childhood, infant and perinatal, England and Wales (series DH3), 1995. London: Office of National Statistics, 1997.

144 Office for National Statistics. Mortality statistics: childhood, infant and perinatal, England and Wales (series DH3), 1994. London: Office of National Statistics, 1996.

145 Office for National Statistics. Mortality statistics: childhood, infant and perinatal, England and Wales (series DH3), 1993. London: Office of National Statistics, 1995.

146 Office for National Statistics. Mortality statistics: childhood, infant and perinatal, England and Wales (series DH3), 1992. London: Office of National Statistics, 1994.

147 Office for National Statistics. Mortality statistics: childhood, infant and perinatal, England and Wales (series DH3), 1991. London: Office of National Statistics, 1993.

148 Office for National Statistics. Mortality statistics: childhood, infant and perinatal, England and Wales (series DH3), 1990. London: Office of National Statistics, 1992.

149 Office for National Statistics. Child mortality (death cohort) tables in England and Wales. London: Office of National Statistics, 2017.

150 Office for National Statistics. Child mortality (death cohort) tables in England and Wales. London: Office of National Statistics, 2016.

151 Office for National Statistics. Child mortality (death cohort) tables in England and Wales. London: Office of National Statistics, 2015.

152 Office for National Statistics. Child mortality (death cohort) tables in England and Wales. London: Office of National Statistics, 2014.

153 Office for National Statistics. Child mortality (death cohort) tables in England and Wales. London: Office of National Statistics, 2013.

154 Office for National Statistics. Child mortality (death cohort) tables in England and Wales. London: Office of National Statistics, 2012.

155 Office for National Statistics. Child mortality (death cohort) tables in England and Wales. London: Office of National Statistics, 2011.

156 Office for National Statistics. Child mortality (death cohort) tables in England and Wales. London: Office of National Statistics, 2010.

157 Office for National Statistics. Child mortality (death cohort) tables in England and Wales. London: Office of National Statistics, 2009.

158 Office for National Statistics. Child mortality (death cohort) tables in England and Wales. London: Office of National Statistics, 2008. 